\title{
Extracellular Matrix Levels Modulate Outgrowths Dynamics in Ovarian Cancer.
}

Sarah Alshehri ${ }^{1^{*}}$, Tonja Pavlovic ${ }^{1^{*}}$, Sadaf Farsinejad ${ }^{1}$, Panteha Behboodi $^{2}$, Li Quan ${ }^{2}$, Daniel Centeno ${ }^{1}$, Douglas Kung ${ }^{1}$, Marta Rezler ${ }^{3}$, Woo Lee ${ }^{1}$, Piotr Jasiński ${ }^{4}$, Elżbieta Dziabaszewska ${ }^{3}$, Ewa Nowak-Markwitz ${ }^{3}$, Dilhan Kalyon ${ }^{2}$, Shang Wang $^{5}$, Mikołaj P. Zaborowski ${ }^{3 \&}$, and Marcin Iwanicki ${ }^{1 \&}$

${ }^{1}$ Department of Chemistry and Chemical Biology, Stevens Institute of Technology, Hoboken, USA

${ }^{2}$ Department of Chemical Engineering, Stevens Institute of Technology, Hoboken, USA

${ }^{3}$ Department of Obstetrics and Gynecology, Poznań University of Medical Sciences, Poznań, Poland

${ }^{4}$ Department of Pathology, Poznań University of Medical Sciences, Poznań, Poland

${ }^{5}$ Department of Biomedical Engineering, Stevens Institute of Technology, Hoboken, USA

* These authors contributed equally.

\&Correspondence: miwanick@stevens.edu or mzaborowski@ump.edu.pl

\section{ABSTRACT}

Ovarian carcinoma $(\mathrm{OC})$ form outgrowths that extend from the outer surface of an afflicted organ into the peritoneum. OC outgrowth formation is poorly understood because there is a limited availability of OC cell culture models to examine the behavior of cell assemblies that form outgrowths. Prompted by immuno-chemical evaluation of extracellular matrix (ECM) components, laminin $\gamma 1$ and collagens, in human tissues representing untreated and chemotherapy-recovered OC, we developed laminin and collagen-rich ECM-reconstituted cell culture models amenable to studies of cell assemblies that can form outgrowths. We demonstrate that ECM promotes outgrowth formation in fallopian tube non-ciliated epithelial cells (FNE) expressing mutant $\mathrm{p} 53^{\mathrm{R} 175 \mathrm{H}}$ and various $\mathrm{OC}$ cell lines. Outgrowths were initiated by cell assemblies that had undergone outward translocation and, upon mechanical detachment, could intercalate into mesothelial cell monolayers. Electron microcopy, optical coherence tomography (OCT) and small-amplitude oscillatory shear experiments revealed that elevating ECM levels increased ECM fibrous network thickness and led to high shear elasticity of ECM environment. These physical characteristics were associated with suppression of outgrowths. Culture environment with low ECM content mimicked viscoelasticity and fibrous networks of 
ascites and supported cell proliferation, cell translocation and outgrowth formation. These results highlight the importance of ECM microenvironments in modulating OC growth and could provide additional explanation of why primary and recurrent ovarian tumors form outgrowths that protrude into the peritoneal cavity containing ascites as opposed to breaking through the basement membrane, invading collagen-dense tissues an intravasating into vasculature.

\section{INTRODUCTION}

Ovarian carcinoma $(\mathrm{OC})$ can arise from the outer mucosa of the epithelial cell surfaces covering reproductive track organs including endometrium ${ }^{1}$, ovary ${ }^{2}$ and the fallopian tubes ${ }^{3}$. Within malignant tissue, transformed epithelial cells can form cell assemblies that protrude, away from the basement membrane and sub-epithelial stroma, toward the peritoneal space ${ }^{4}$. These cell assemblies can detach, transit into the abdominal cavity and, through superficial attachment and intercalation into mesothelial cell layers, contribute to the spread of the peritoneal disease ${ }^{5,6}$. After surgery and chemotherapy, surviving tumor cells continue to form outgrowths leading to recurrent disease characterized by the enrichment of detached tumor clusters in the peritoneal cavity often containing ascites, the inflammatory fluids rich in various mitogenic factors including ECM molecules ${ }^{7}$. Thus, investigating the processes associated with OC outgrowth formation would provide important information about the mechanisms of disease progression.

ECM is a molecular scaffold rich in numerous glycosylated proteins including laminin $\gamma 1^{8}$ and collagens ${ }^{9}$ that, through interaction with cell surface receptors ${ }^{10}$, provide mechanical $^{11-14}$ and biochemical ${ }^{15,16}$ cues to regulate tissue development ${ }^{17,18}$ and malignant progression ${ }^{19,20}$. Recent histologic examination of OC outgrowths protruding from the fallopian tube demonstrated enrichment of laminin $\gamma 1$ within tumor cells forming outgrowths ${ }^{21}$. Consistent with these findings, we previously reported ${ }^{22}$ that both detached human fallopian tube none-ciliated epithelial (FNE) cells, expressing various mutant p53 proteins and patient-derived ovarian tumor 
cell clusters deposited on their cell surfaces, fibronectin, an ECM molecule that supports tumor survival and cell-ECM-cell adhesion. Most recent studies ${ }^{23}$ using second harmonic visualization of outgrowths arising in the fallopian tube revealed the presence of yet other ECM components, the collagens. Collagen molecules were found to form fibrillar "wavy" networks beneath basal surfaces of transformed epithelium. Taken together these data provoke questions of whether and how tumor microenvironments, such as ECM, regulate the behavior of cell assemblies that form outgrowths.

Reconstitution of cell cultures with a laminin and collagen-rich basement membrane extract, Matrigel $(\mathrm{MG})$, has become a standard approach $^{24}$ to study the role of ECM in cellular growth $^{25}$, migration ${ }^{26}$ and death ${ }^{27}$. Several recent studies ${ }^{28-31}$ employed MG to grow OC cells to evaluate their response to treatments in the context of three-dimensional (3D) tumoroid structures that resemble malignant tissue organization more closely than do two-dimensional (2D) cell cultures. While these studies provided a static view of tumor cells with ECM reconstitution, the contribution of ECM microenvironments to the dynamic process of OC growth remains unknown.

In this report we have performed immuno-chemical evaluations of laminin $\gamma 1$ and collagens in $\mathrm{OC}$ representing disease progression before and after chemotherapy. As in of OC tumorigenesis from the fallopian tube ${ }^{21}$, we found that laminin $\gamma 1$ was predominantly associated with tumor cells, whereas collagens formed fibrillar networks within the surrounding microenvironment. These studies indicated that ECM deposition within tumor cells and adjacent connective microenvironments contributes to OC outgrowth dynamics. Motivated by these results we deployed laminin and collagen-rich (MG) ECM-reconstituted 96-well-based suspended or adhered cell culture models to study dynamics of OC cell assemblies using livecell imaging and quantitative image processing of cell proliferation and translocation. We found that ECM reconstitution of suspended cell clusters induced outgrowths in FNE cells expressing 
mutant $\mathrm{p} 53^{\mathrm{R} 175 \mathrm{H}}$, the cells-of-origin for a proportion of OCs, and genetically distinct and wellestablished OC cells lines. Live-cell recordings revealed that outgrowths were initiated by cell assemblies that had undergone outward translocation and, upon mechanical detachment, could intercalate into mesothelial cell monolayers, the most frequent metastatic sites for OC.

Increased ECM content altered fibrous network thickness and viscoelastic characteristics of the microenvironment, which led to the suppression of cell proliferation, directional cell translocation and outgrowths. Furthermore, ECM reconstituted culture that supported outgrowths, cell proliferation and cell translocation mimicked viscoelastic properties of ascitic fluids. Our results are consistent with a model whereby low-elasticity ECM microenvironments support cell proliferation and translocation of cell assemblies that form outgrowths. Our data could partially explain why primary and recurrent OCs form outgrowths that protrude into ascites, as opposed to breaching underlying collagen-dense stroma and intravasating into nearby vasculature.

\section{RESULTS}

\section{ECM deposition is associated with OC outgrowths.}

Detached OC cell clusters deposit ECM on cell surfaces to support survival and cell-ECM-cell adhesion $^{22}$. Pathologic examination of early tumors indicated that the sources of detached OC cell assemblies are malignant outgrowths that protrude into the peritoneal space from the outer mucosal surface of the fallopian tube ${ }^{4}$. To examine whether OC cell assemblies are associated with ECM deposition, we used a combination of immunohistochemistry (IHC) for laminin $\gamma 1$ and picrosirius red stain that decorates dense fibrillar collagens (I, II and IV) $)^{32}$ contained within connective tissue and the basement membrane. These approaches revealed that PAX8-positive (marker of $\mathrm{OC}$ ) cell assemblies formed outgrowths protruding from the surface of the human ovary (Figure $1 \mathrm{~A}$ ), or in a patient-derived xenograft model (PDX), from the omentum (Figure 1 E). OC outgrowths contained laminin $\gamma 1$ (Figure 1A, I, red arrows) and, to a much lesser degree, collagens (Figure 1C, G, black arrows). In the same tissue sections, we observed that 
adjacent extra tumoral connective space was low in laminin $\gamma 1$ and contained dense fibrillar "wavy" collagen networks (Figure 1C, G, green arrows). Image analysis of laminin $\gamma 1$ and collagens expression revealed significant difference in localization of these ECM molecules. Laminin $\gamma 1$ was enriched within tumors (Figure 1B, F), whereas collagens were mostly enriched within extra-tumoral space (Figure 1D, H). Taken together, these results indicate that OC outgrowths are associated with at least two ECM microenvironment factors, laminin $\gamma 1$ and collagens.

\section{Laminin $\gamma 1$ and collagens are associated with OC before and after chemotherapy.}

Some patients develop recurrent disease characterized by the presence of detached tumor clusters within ascitic fluids accumulating in the peritoneal cavity ${ }^{7}$. These clinical observations indicate the that similarly to primary tumors, tumors that have recovered from chemotherapy are associated with laminin $\gamma 1$ and collagens -rich ECM microenvironments. Thus, we wanted to evaluate ECM deposition in tumors recovered from chemotherapy. Pathologic examination of PAX8-positive primary tumors in the ovary (Figure 2A) and matched omental metastases representing tumors before and after neo-adjuvant chemotherapy revealed similar laminin $\gamma 1$ and collagen deposition (Figure 2B-E). These data indicate that OC recovery from chemotherapy is associated with the presence of laminin $\gamma 1$ and collagens ECM microenvironments and support the idea that laminin- and collagen-rich ECM can contribute to OC outgrowth dynamics before and after chemotherapy.

\section{ECM reconstitution of suspended OC cell clusters promotes outgrowths.}

Laminins (including laminin $\gamma 1$ ) and collagens (including collagen IV) represent scaffold components of the basement membrane ${ }^{33,34}$ and of the reconstituted basement membrane extract, $\mathrm{MG}^{35}$. To partially mimic ECM microenvironments associated with OC, we tested whether MG supports formation of OC outgrowths in suspended cell cultures. We initially chose to examine $M G$ effects on outgrowth formation in FNE cells expressing mutant p53 ${ }^{\mathrm{R} 175 \mathrm{H}}$ (FNE- 
m-p53). We chose this cell line, because; (1) fallopian tube non-ciliated cells, with mutations in the TP53 gene, are thought to be precursors of OC and an initial source of outgrowths that can disseminate into the peritoneum; and (2) we have recently demonstrated ${ }^{22}$, using this cell line, that expression of m-p53 in FNE cells induces acquisition of transformed phenotypes associated with OC progression including survival of detached cellular clusters and mesothelial intercalation $^{22}$. Here, we examined outgrowth forming capabilities of FNE-m-p53 or FNE cells expressing empty plasmid. First, we stimulated cell assembly formation by clustering cells in suspension for 24 hours in single wells of low-adhesion 96-well plates (Figure 3A). Second, to mimic laminin and collagen-rich ECM microenvironments, we reconstituted cell assemblies with media containing 2\% MG (Figure 3A). We chose this ECM reconstitution condition because we have previously reported that $2 \%$ MG supported growth ${ }^{27}$ of suspended breast cancer cell cultures. Live-cell microscopy revealed that, FNE-m-p53 but not FNE expressing empty plasmid significantly expanded and formed outgrowths initiating from the cell assemblies that, over time, began to extend beyond the cell cluster (Figure 3B-C, and MOVIE 1). We observed that FNEm-p53 cell clusters formed outgrowths with average frequency of $30 \%$, whereas no outgrowths were observed in cell clusters composed of FNE cells expressing empty plasmid or FNE-m-p53 cultured without MG (Figure 3D). These data are consistent with the crucial role of ECM deposition in supporting outgrowth development by FNE-m-p53 cells.

The mode of intraperitoneal dissemination is a common feature among different types of OCs such as serous and non-serous types ${ }^{6}$. Therefore, we wanted to evaluate whether OC cell lines, representing other tumor types, form outgrowths with $2 \%$ MG reconstitution. We examined additional OC cell lines, Hey-A8 and Tyk-nu representing non-serous and likely serous OC models as defined by Domcke et al., ${ }^{36}$. As shown in Figure 3 E, reconstitution of Hey-A8 (MOVIE2) or Tyk-nu (MOVIE3) cellular clusters with 2\% MG promoted outgrowth formation. The average percentage of outgrowth formation among Hey-A8 and Tyk-nu cell clusters varied from $50 \%$ to $10 \%$, respectively (Figure 3 E). Our data suggest that ECM deposition contributes to the 
formation of outgrowths in some suspended cell clusters representing fallopian tube cells expressing cancer driving mutant p53 protein, serous and non-serous OC cells.

\section{Detached outgrowths clear mesothelial monolayers.}

OC outgrowths can ultimately detach and intercalate into mesothelial surfaces of abdominal organs, such as the ovary ${ }^{1}$. So, we wanted to know whether OC outgrowths, that develop in ECM-reconstituted cultures, can intercalate into mesothelial cell layers. To do this, we examined Hey-A8 MG-reconstituted cell cultures, as these formed the longest protruding outgrowths that could be most easily dissociated from cell clusters under a dissecting microscope. We mechanically separated Hey-A8 outgrowths from cell clusters and co-cultured both entities on the surface of mesothelial cell monolayers that express green fluorescent protein (GFP) (Figure 4A). Of note, we observed that, upon detachment, outgrowths changed their shapes from "rod-like," to a spherical structure resembling free-floating OC spheroids commonly found in malignant ascites. To examine the outgrowths 'and cell clusters' abilities to invade mesothelial monolayers, we used a mesothelial clearance assay ${ }^{37}$ which relies on measuring the area of a "hole" made by OC cells in a mesothelial cell monolayer ${ }^{38}$. Hole formation is visualized by the time-dependent exclusion of GFP fluorescence reporting on mesothelial cell migration beneath an OC cell assembly. By using this assay, we observed that detached outgrowths induced mesothelial clearance while cell clusters, from which the outgrowths were separated, showed less clearance (Figure 4B-C). The observed results could be explained by the possible difference in viability between cells contained within outgrowths and cell clusters. Spatial examination of cell viability, within cellular structures, using propidium iodide $(\mathrm{PI})$, a fluorescent dye permeating only dead cells, revealed that many dead cells were localized to the cell clusters whereas outgrowths showed minimal PI incorporation (Figure 4DE), which was indicative of a good cell viability. These data are consistent with the hypothesis that $\mathrm{OC}$ cell assemblies forming outgrowths are viable and upon detachment can intercalate into mesothelial monolayers. 


\section{Increase of ECM deposition inhibits outgrowths.}

Up to this point, our data provide evidence that ECM deposition induces OC outgrowths that can intercalate into mesothelial cell layers. Next, we wanted to gain more information about the role of ECM in the regulation of OC outgrowths. Hence, we examined whether modulation of ECM content influences outgrowth formation. We compared outgrowth formation capability of suspended FNE-m-p53 and Hey-A8 cell clusters reconstituted with $2 \%$ or $25 \%$ MG. As expected, reconstitution of both cell types with $2 \%$ MG led to outgrowth formation, however, 25\% MG culture conditions significantly attenuated outgrowth formation (Figure 5A-B), indicating that increasing ECM content suppresses outgrowths. We noticed that under $25 \%$ MG conditions, both FNE-m-p53 and Hey-A8 cells clusters appeared to be moderately, but significantly, smaller when compared to $2 \%$ MG reconstitution conditions (S. Figure 1A, B). Of note, as opposed to FNE-m-p53 cells, Hey-A8 cells cultured in 25\% MG were less compact and more disorganized (Figure 5B, inset), suggesting that elevating ECM levels may disrupt HeyA8 cell-cell adhesion. Nevertheless, these data indicated the possibility that increasing ECM deposition affects cell proliferation. To explore this possibility, we took advantage of cell culture assay in which cell monolayers are overlayed with $M \mathrm{MG}^{39}$ (S. Figure 1C), mimicking conditions of ECM encapsulation. We integrated this assay with live-cell imaging of FNE-m-p53 and HeyA8 cells expressing GFP or mKO2, respectively. As shown in S. Figure 1D, E, MOVIE4 and MOVIE5 encapsulation of FNE-m-p53-GFP or Hey-A8-mKO2 cells with $25 \%$ MG significantly decreased cells proliferation. These results were also supported by the data indicating that $25 \%$ MG reconstitution of suspended Hey-A8 cell cultures significantly reduced the number of cells undergoing DNA replication, as assessed by 5-ethynyl-2'-deoxyuridine (EdU) incorporation assays (S. Figure 1 F). We chose to examine EdU incorporation in Hey-A8 cells because these cells formed significantly larger structures when compared to FNE-m-p53 cell clusters (S.

Figure 1A-B), making EdU incorporation analysis more robust. Our results are consistent with 
the idea that ECM levels modulate viscoelastic proprieties of OC cell environment to control OC cell proliferation and outgrowth formation.

\section{Elevation of ECM content leads to the formation of thick fibrillar networks}

Our IHC examination of tumors indicated that OCs do not invade into thick collagen fibrous networks associated with tissue that surrounds tumors (Figure 1). Increasing ECM leads to suppression of outgrowths and cell proliferation (Figure 5 A, B, S. Figure 1). These results motivated us to examine whether elevating ECM increases fibrous network thickness. To do so, we used a combination of electron microscopy and optical coherence tomography (OCT). As shown in Figure 5C scanning electron microscopy (SEM) revealed qualitative differences in ECM networks formed in $2 \%$ and $25 \%$ MG. We observed that network fibers formed in $25 \%$ MG were larger and appeared to be thicker compared to network fibers formed in $2 \% \mathrm{MG}$. These results suggest the possibility that elevation of MG concentration leads to the formation of larger ECM fibers. For quantitative evaluation of ECM fibers, we imaged the MG samples with OCT, where the endogenous optical contrast represents the microstructure of the sample. The OCT B-scan images revealed a higher contrast from the ECM contents with larger high-intensity clusters in the $25 \%$ MG compared with the $2 \%$ MG (Figure. 5D), suggesting a relatively larger ECM fiber size in the $25 \%$ MG. Also, the representative OCT depth-resolved intensity profiles show a generally larger spatial period of intensity fluctuations in the $25 \%$ MG (Figure. 5E), which can be clearly seen with the relatively more low-frequency components from the corresponding amplitude spectra (Figure. 5F). This feature in the spatial frequency was quantified with the decay coefficient from an exponential fit of the local peaks in the amplitude spectra (Figure. 5F), where the higher exponential decay coefficient in $25 \%$ MG indicates relatively stronger low-frequency components, suggesting thicker ECM fibers in $25 \% \mathrm{MG}$. These results led us to think that increase in ECM fiber thickness results in elevation of OC cell culture elasticity. 


\section{ECM levels modulate viscoelasticity of OC culture microenvironments.}

Based on the data demonstrating larger size of ECM fiber networks formed in $25 \%$ MG cultures, we wondered whether changing concentration of ECM modulates a culture's viscoelasticity i.e., time dependent elasticity and viscous behavior of the MG cell culture ${ }^{40}$.

We characterized the linear viscoelastic material functions of cell culture media containing $2 \%$ or $25 \%$ MG. This analysis revealed, that under $25 \%$ MG reconstitution of culture media, the loss modulus, G", values representing viscous behavior (indicative of the energy dissipated as heat when the culture media is deformed under small-amplitude oscillatory shearing), were within a narrow range of 0.5 to $0.9 \mathrm{~Pa}$ in the frequency range of 0.1 to $10 \mathrm{~s}^{-1}$ and thus were not sensitive to the frequency of rotation (Figure 6A). Under similar conditions the storage modulus, G', values representing elasticity, i.e., indicative of the energy stored as elastic energy during the deformation of the culture media, were within the range of 3.5 to 4.5 Pa. The storage modulus, G', data were thus also not sensitive to the frequency of rotation (Figure 6A). The loss moduli, G", values of samples with $2 \%$ MG ranged between 0.05 to 0.1 Pa while the storage modulus, G', values of these $2 \%$ MG samples were between 0.09 to 0.3 $\mathrm{Pa}$ (Figure 6B). The storage moduli of $25 \% \mathrm{MG}$ reconstitution culture media were significantly higher than the loss moduli (G' > G"), and the ratio of G'/G' (tan $\delta$ ) was below 1 (Figure 6C). On the other hand, $2 \%$ Matrigel reconstitution cultures produced tan $\delta$ values greater than 1 (Figure 6D) indicating less elastic nature of the $2 \%$ versus the $25 \%$ Matrigel. These results suggest that elasticity of the $25 \%$ MG reconstitution media is significantly higher than that of $2 \%$. Interestingly, measurement of loss and storage moduli of peritoneal ascites isolated from a patient with ovarian cancer, revealed close similarity to G" and G' values and $\tan \delta$ observed in the $2 \%$ MG cultures (Figure 6E, F), indicating that ascites represent gels with low strength values. These results motivated us to examine whether ascites form extracellular fibrous networks. Scanning electron microscopy evaluation of ascitic fluids revealed the presence of 
extracellular fibers that were also associated with tumor cells (Figure 6G-I). These results are consistent with the hypothesis that ECM-rich microenvironments, with low shear viscosity and elasticity, such as $2 \%$ MG or ascitic fluids, support outgrowths formation.

\section{Increasing ECM deposition suppresses directional translocation.}

Based on the data demonstrating that outgrowths were initiated by cell assemblies that translocated outside the cellular structure (Figure 3, MOVIE1-3) and increasing ECM concentration suppressed outgrowths, decreased cell cluster size, and cell proliferation (Figure

5 and S. Figure 1), we hypothesized that high ECM levels also suppress cell translocation. To track cells encapsulated by ECM, we overlayed FNE-m-p53-GFP and Hey-A8-mKO2 cells grown on $2 \mathrm{D}$ surfaces with $2 \%$ or $25 \%$ MG (S. Figure 1 C). Similarly, to $3 \mathrm{D}$ culture, under this condition FNE-m-p53-GFP cells-maintained cell-cell adhesion (S. Figure 1G), forming welldefined and coherent colonies. However, as opposed to 3D conditions, Hey-A8 cells remained as single cells (S. Figure 1G), making it difficult to track cell translocation within the group of cells. Thus, we analyzed cell translocation of FNE-m-p53-GFP cells encapsulated with different MG concentrations. To quantify the dynamics of cell translocation over time, we integrated TrackMate with Trajectory classifier (both ImageJ plug-ins) to compute mean squared displacement of individual cell translocations and based on this information, we derived classified cell trajectories. Using this approach, we observed that reconstitution of FNE-m-p53 with $2 \%$ MG resulted in directed cell translocation, as visualized by the presence of sustained tracks that extended, over time, in $53 \%$ of classified cell trajectories (Figure 7A, C and MOVIES 6). Increasing MG content to $25 \%$, significantly reduced classified cell trajectories (Figure $7 \mathrm{~B}, \mathrm{C}$ and MOVIE 7), indicating that increase in ECM deposition suppresses directional cell translocation within group of cells. These results are consistent with the idea that elevation of ECM levels increases elasticity of OC microenvironments and restricts outgrowth development through the suppression of cell proliferation and directional cell translocation. 


\section{DISCUSSION}

In tissue samples representing human $\mathrm{OC}$, IHC of laminin $\gamma 1$ and picrosirius based detection of fibrillar- and basement membrane associated collagens revealed that OC outgrowths were associated with two distinct ECM microenvironments. The first ECM microenvironment was observed among carcinoma cells and contained laminin $\gamma 1$, with low levels of collagens. The second showed low laminin $\gamma 1$ extra-tumoral stroma that contained dense collagen fibers. These results prompted us to use a laminin- and collagen-rich reconstituted tissue culture approach to study spatial and temporal evolution of OC outgrowths. Laminin- and collagen-rich $\mathrm{MG}^{35}$ reconstitution of $\mathrm{OC}$ cell cultures was used in combination with live-cell imaging, scanning electron microscopy, optical coherence tomography and rheology to examine the contribution of ECM to OC cell-assembly dynamics. Live-cell imaging studies demonstrated that ECM reconstitution of suspended cell cultures, representing various OC types, promoted formation of outgrowths that, upon detachment, intercalated into mesothelial cell layers. We observed outgrowth formation in multiple genetically distinct OC cell lines including FNE-m-p53. ECM reconstitution did not promote outgrowths in "normal" nontransformed FNE cultures, indicating that outgrowth formation is associated with transformed phenotypes of the cells-of-origin. OC outgrowths were initiated by cell assemblies that had undergone outward translocation. Increasing ECM deposition led to increased elasticity of OC cell culture media, suppressed proliferation, decreased directional cell translocation and suppressed outgrowths. Based on these results we propose a model in which OC cells favor low elasticity ECM microenvironment to divide, translocate and form outgrowths that extend into the peritoneal cavity (Figure 7E). Our data potentially explain why genetically distinct OC types are superficially invasive and prefer to grow away from ECM dense stroma and form outgrowth protrusions extending into the low elasticity environment of the peritoneal ascites. 
Recent IHC evaluation of OC within the fallopian tube revealed enrichment of laminin $\gamma 1$ chain ${ }^{21}$, a component of the heterotrimeric laminin $\alpha \beta \gamma$ molecule ${ }^{41}$. Consistent with these studies, we found that cancer cells representing human OC outgrowths protruding from the ovary and omental metastases were enriched in laminin $\gamma 1$. In contrast to tumor cells, the adjacent stroma displayed low levels of laminin $\gamma 1$ deposition, highlighting a possible involvement of $\gamma 1$ chain containing laminins in the development of cell-ECM-cell adhesion among tumor cells forming outgrowths. Laminins promote formation of basement membranes that support epithelial cell proliferation and collective cell movements during tissue development ${ }^{42,43}$. We speculate that formation of a sufficient ECM adhesion among OC cells could contribute to tumor expansion and cell translocation leading to initiation of malignant outgrowths. To support this speculation, we provide evidence that reconstitution of OC cell clusters with ECM promoted proliferation, directional cell translocation and outgrowths.

We observed a dense and thick collagen fiber network enriched within extra-tumoral spaces. These results were consistent with previous studies demonstrating collagen fiber localization beneath $\mathrm{OC}$ outgrowths originating from the fallopian tube ${ }^{23}$ and stroma surrounding metastatic omental implants ${ }^{44}$. Collagen fiber formation occurs when collagen concentration increases ${ }^{45}$, indicating that extra-tumoral fibrils reflect the presence of highly elastic ECM. Metastatic OC superficially invades mesothelial layer of peritoneal organs ${ }^{6}$ and consistently we observed that OC tumors injected into mouse peritoneum colonized surfaces of the omentum and formed superficial outgrowths extending into the peritoneal cavity, with minimal invasion of underlying stroma, including fibrillar collagens. Based on these results, we speculated that dynamic evolution of OC outgrowths is supported by low ECM content microenvironments. Thereby, we evaluated the dynamics of cell translocation and outgrowth formation under low (2\%) and high - (25\%)-MG content conditions. We demonstrated that cell translocation, cell division and outgrowth formation was more robust under $2 \%$ MG culture conditions, indicating a 
possibility that high content of ECM surrounding OC cell clusters provides a physical barrier that suppresses OC progression. To directly test this possibility, we overlayed cell monolayer cultures with media containing $2 \%$ or $25 \%$ MG and observed that $25 \%$ MG significantly suppressed proliferation and restricted directional cell translocation. The correlations between cell proliferation, cell translocation and outgrowth formation are consistent with the ability of recurrent disease to form new metastases and support the idea that OC growth drives metastasis.

Recent studies demonstrate that increasing concentration of collagen restricted single cell translocation from the cell clusters, as measured by mean square displacement of the tracked trajectories ${ }^{46}$, and targeting fibroblasts that make collagens results in reduced collagen deposition and increased OC cluster dispersal ${ }^{44}$. Furthermore, 2D studies of the effects of ECM concentration on cell motility have shown optimal ECM concentrations that supported speed of cell migration. Concentrations below and above these optimal values did not stimulate fibroblast migration to same level ${ }^{47}$. Our studies support this relationship because OC cells that were reconstituted in a high concentration of ECM showed reduction in cell translocation and outgrowth formation. This could be explained by optimal integrin-complex turnover rates under appropriate (likely low) ECM concentrations. It is well established that high concentrations of ECM leads to strong adhesion ${ }^{47}$ and low adhesion turnover ${ }^{48}$. Therefore, we speculate that microenvironments with low ECM content support formation of adhesion complexes that are highly dynamic and, thus, promote cell translocation. In contrast, attachment of OC cells to extratumoral and dense ECM microenvironments would lead to strong adhesion and cancer cell "immobilization". This speculation is supported by observation demonstrating larger size of ECM fibrous networks and decreased directional cell translocation under high-ECM reconstitution conditions. This could explain why OC outgrowths rarely invade collagenous stroma to intravasate into vasculature.

Varying ECM content modulates viscoelastic characteristics of the microenvironment and could affect cell behavior ${ }^{49}$, including acquisition of transformed phenotypes ${ }^{50}$. Using 
electron microscopy, optical coherence tomography and rheometric analysis of ECMreconstituted cultures, we provide evidence that $2 \% M G$ reconstitution results in the formation of thin (as compared to $25 \%$ MG cultures) extracellular fibrous networks and low-elastic properties of cultures, whereas $25 \%$ ECM reconstitution led to the development of thick fibrous networks and elastic properties in cell culture media, a characteristic of more solid environment. Our data demonstrated that ECM concentrations that maintain low elasticity of tissue culture support outgrowths, through cell proliferation and directional cell translocation. Thus, it is conceivable to propose that ECM deposited on the surface of OC tumor cells creates a low-elasticity microenvironment that provides enough adhesion and traction force to support, cell division and cancer cell directional translocation. Increasing deposition of ECM within tumor microenvironment would increase adhesiveness, and suppress cell proliferation, cell translocation and outgrowth formation (Figure 7E). Our model is consistent with the clinical observations that $\mathrm{OC}$ peritoneal dissemination is associated with tumor outgrowths that protrude into the peritoneal cavity ${ }^{4,21}$ where ascites, representing low-elasticity microenvironment ( as oposed to solid collagen-rich tissues), are enriched with ECM molecules ${ }^{7,51}$. To support our model, we provide evidence that shear moduli values of $2 \%$ MG cultures resembled ascites enriched with ECM fibrous networks that surrounded carcinoma cells.

OC outgrowths continue to evolve after chemotherapy because detached tumor cell clusters are present within the peritoneal cavity fluids of patients with recurrent disease ${ }^{7}$. We demonstrate that $\mathrm{OC}$ cells that have recovered from chemotherapy are in direct contact with ECM deposited within tumors and extra-tumoral space. Thus, our ECM-reconstitution model of OC outgrowths offers a clinically relevant approach to examine evolution of recurrent phenotypes, such as outgrowths dynamics, associated with the disease. The implementation of live-cell imaging, scanning electron microscopy, optical coherence tomography and rheology provided important insights into the role of physical characteristics of OC cell microenvironments and their contribution to OC outgrowth dynamics. 


\section{METHODS}

\section{Cell Culture}

Hey-A8 (obtained from Dr. Sumegha Mitra laboratory, University of Indiana) and Tyk-nu (obtained from Dr.Joan Brugge laboratory, Harvard Medical School) cells were cultured in a 1:1 ratio of Medium 199 (Gibco) and MCDB105 (Sigma) supplemented with 5\% fetal bovine serum (Gibco), 1\% Penicillin-Streptomycin (Gibco) and $50 \mu \mathrm{g} / \mathrm{ml}$ plasmocin prophylactic (InvivoGen). FNE cells (obtained from Dr.Tan Ince laboratory, Weil Cornel Medicine , New York) were cultured in 1:1 ratio of Media 199 (HiMedia) and DMEM/F12 (HiMedia), 3\% HI-FBS (Corning), 1\% PenicillinStreptomycin (Sigma), $0.5 \mathrm{ng} / \mathrm{ml}$ of 17 beta-estradiol (US Biologicals), $0.2 \mathrm{pg} / \mathrm{ml}$ of triiodothyronine (Sigma), $0.025 \mathrm{~g} / \mathrm{ml}$ all-trans retinoic acid (BeanTown Chemicals), $20 \mathrm{mg} / \mathrm{ml}$ of insulin (Sigma), $0.5 \mathrm{ng} / \mathrm{ml}$ of EGF (Peprotech), $0.5 \mu \mathrm{g} / \mathrm{ml}$ hydrocortisone (Sigma), $25 \mathrm{ng} / \mathrm{ml}$ of Cholera Toxin (Calbiochem, USA) and $50 \mu \mathrm{g} / \mathrm{ml}$ plasmocin prophylactic (InvivoGen). Cell cultures were tested for the presence of mycoplasma every 3-6 months using the Uphoff and Drexler detection method ${ }^{52}$.

\section{ECM Reconstitution}

100 cells per well were seeded on ultra-low attachment 96-well plates (Corning). Plates were spun at 900 RPM for 3 minutes and incubated until the following day. The next day, on ice and using prechilled pipette tips, $100 \mu \mathrm{l}$ of $4 \%$ or $50 \%$ Matrige $\AA^{\circledR}(\mathrm{MG})$ (Becton-Dickinson) was added to each well containing $100 \mu \mathrm{l}$ of media and cell clusters (final culture volume was $\sim 200 \mu \mathrm{L}$ ).

\section{Processing of Cell Clusters for Immunohistochemistry (IHC).}

Cell clusters were fixed in 4\% paraformaldehyde solution (Sigma) then dehydrated in ethanol. Histogel (Thermo Scientific) was liquefied, and cell clusters were mixed with Histogel and left to solidify in a biopsy cassette. Histogel blocks were fixed in $10 \%$ neutral buffered formalin overnight then dehydrated in ethanol and cleared in xylene. Processed Histogel blocks were then 
embedded in paraffin and stored at $-20^{\circ} \mathrm{C}$ until time of sectioning. Paraffin blocks were sectioned at a thickness of $5 \mu \mathrm{m}$ using a microtome (Leica).

\section{IHC}

Formalin-Fixed Paraffin-Embedded (FFPE) patient-derived xenograft tissue (DF118-PDX) is a kind gift from Dr. Ronny Drapkin, University of Pennsylvania. Sections were deparaffinized in two xylene changes for 10 minutes each, then sections were de-hydrated in a graded series of ethanol $(100 \%, 90 \%, 70 \%, 50 \%) 5$ minutes each, and washed in ultrapure water for 5 minutes. The ABC kit; Vectastain Elite (Vector Laboratories) was used, per the manufacturer's recommendation. The primary antibodies used were anti-PAX8 (1:1000; \#10336-1-AP; Protein tech.), anti-LAMC1 (1:500; \#HPA 001909; Sigma Aldrich). Peroxidase substrate (ImmPACT DAB; \#SK-4105; vector laboratories) was used to develop and visualize staining under the microscope (Nikon ECLIPSE Ci-L using Nikon DS-Fi3 camera). The slides were rinsed in water, dehydrated in a series of ethanol solutions of increasing concentrations, until $100 \%$ ethanol was reached, then cleared in xylene, mounted with a non-aqueous mounting medium (\#H-5000; Vector Laboratories), sealed using clear nail polish and left to dry before imaging. Collagen staining was performed using a Picrosirius red staining kit (\#24901; Polysciences, Inc.) per the manufacturer's recommendation. Deparaffinization and hydration were followed by 1-hour staining in Picrosirius Red, washing in hydrochloric acid, dehydration, and mounting as described above.

\section{IHC Image Acquisition}

Stained slides were scanned using an Olympus IX83 microscope equipped with a DP80 color camera and CellSens software. Based on scanned slides, ROls were identified and recaptured using a Nikon Eclipse equipped with DS-Fi3 color camera and NIS Elements D software. Images were saved as TIFFs for further processing. 


\section{IHC Image Processing}

As previously described ${ }^{53}$, RGB images were converted, using a weighted RGB conversion option in $\mathrm{FIJI}^{54}$ (Fiji is just ImageJ). software, to an 8-bit grayscale range representing values between white (0) and black (255). To make backgrounds white, images were inverted and multiple ROls representing tumor, stroma or areas without tissue were selected. Mean gray values (values between 0-255) were calculated for each ROI and plotted.

\section{Live-Cell Imaging of Cell Clusters}

Ultra-low adhesion 96-well plates containing ECM reconstituted cell cultures were placed within a BioTek LionHeart FX long-term imaging chamber equipped with enclosed optics, temperature and gas exchange controls. Individual cell clusters were imaged for up to 7 days with indicated time intervals. Multiple XYZ planes of cell clusters were acquired simultaneously.

\section{Quantification of Outgrowth Protrusion Frequency}

To quantify the frequency of outgrowth protrusions by cell clusters representing different OC cell lines, different laboratory members set up at least 30 suspended cell cluster cultures reconstituted with $2 \%$ MG. Outgrowths were defined as distinct cell populations that appeared outside of the main cell cluster. Scoring was done using a Nikon 2000 tissue culture microscope with a 10X objective.

\section{Pathology Scoring of Laminin $\gamma 1$ Expression in Human Tumors}

Intensity profiles of IHC were qualitatively scored as strong (+3), moderate $(+2)$, weak $(+1)$ and negative $(0)$. IHC results were recorded by a pathologist using $\mathrm{H}$-scores. $\mathrm{H}^{\text {score }}=\left(\%\right.$ cells $\left.{ }^{+} 3 \times 3\right)+$ $\left(\%\right.$ cells $\left.{ }^{+} 2 \times 2\right)+\left(\%\right.$ cells $\left.{ }^{+} 1 \times 1\right)+(\%$ cells $0 \times 0)$. Data are represented as dot plots of $\mathrm{H}$-scores for matched biopsy. Since the data falls into a category of a not-normal distribution, a (nonparametric) Wilcoxon rank-sum test was used to calculate significant differences between pre- and post-therapy specimens. 


\section{Ascites and solid carcinoma tissue collection}

Ascitic fluids were collected from patients with ovarian cancer either by paracentesis under local anesthesia or at the very beginning of a surgery. Ascites fluids were centrifuged at $1,100 \times \mathrm{g}$ for $10 \mathrm{~min}$ at room temperature to remove cell clusters, aliquoted and stored in $-80 \mathrm{C}$ until analysis. All solid tissue collections were performed during surgery. All procedures performed in studies involving human participants were in accordance with the ethical standards of the institutional and national research committee and with the 1964 Helsinki declaration and its later amendments or comparable ethical standards. Patients provided a signed informed consent, approved by the Ethics Review Board of Poznań University of Medical Sciences (Consent No 737/17).

\section{Mesothelial clearance assay}

Mesothelial cells were plated on glass-bottom dishes (Mat-TEK Corporation), which had been coated with $5 \mu \mathrm{g} / \mathrm{ml}$ of fibronectin (Sigma, USA) and/or collagen I (Sigma, USA). Cells were maintained in culture until confluent (48 hours after plating). In co-culture experiments, cell clusters and outgrowths were added to a confluent mesothelial monolayer expressing green fluorescent protein (GFP), allowed to attach, and imaged for up to 120 hours using a LionHeart FX (BioTek) inverted Motorized Widefield Fluorescence Microscope. Only cellular clusters or outgrowths that remained attached during the experiment were used for quantification.

Mesothelial clearance was quantified as previously described ${ }^{46}$.

\section{Determination of Outgrowth Length and Cellular Structure Area}

GEN5 image analysis software (BioTek) was used to quantify outgrowth length and ImageJ to analyze cellular cluster size. Before quantification, a series of bright field images, representing multiple $X Y Z$ planes of a single cellular structure were collapsed to generate maximal projections of cell cluster and protruding outgrowth. Outgrowth protrusion was identified as a structure that extended beyond the cell cluster, and a line was drawn from the tip across the longer axis of the outgrowth, terminating at the junction between outgrowth protrusion and cell cluster. The size of 
the cellular structure was calculated using an in-house ImageJ macro. Briefly, binary masks were created to separate a single cellular structure from the non-cellular background.

\section{EdU incorporation assay}

MG reconstituted Hey-A8 cells were incubated with 10 mM Click-iTÒ EdU [Thermo Fisher Scientific, catalog number C10340] for 4 hours. Cells were fixed with 4\% PFA for 1 hour at room temperature, embedded into HistoGel and sectioned. Sections were permeabilized with 1X PBS containing $0.5 \%$ Triton-X. Residual Triton-X was removed by washing twice with $1 \mathrm{X}$ PBS supplemented with $3 \%$ BSA. To label the incorporated EdU, sections were incubated in the dark for 30 minutes with Click-iT reaction cocktail prepared fresh ( $<15$ minutes before labeling). ClickiT reaction cocktail was then removed, and sections were washed with 1X PBS. To label total cell nuclei, sections were incubated in the dark with $10 \mu \mathrm{g} / \mathrm{mL}$ Hoechst 33342 in 1X PBS for 30 minutes. After washing out the Hoechst dye with 1X PBS, sections were mounted with aqueous mounting media and sealed by coverslips. Sections were imaged on ZEISS LSM -880 confocal microscope using a 10X objective. Images of EdU-labeled nuclei were acquired using far-red laser illumination $(I=647 \mathrm{~nm})$ and total nuclei were captured by violet blue laser illumination $(I=$ $405 \mathrm{~nm})$. Images were analyzed using open-sourced software $\mathrm{FIJI}^{54}$.

\section{Cell proliferation, cell motion imaging and quantifications}

Monolayers of FNE-m-p53 or HEY-A8 cells expressing GFP or mKO2, respectively were overlayed with MG. Time-lapse imaging was performed on a BioTek Lionheart FX automated microscope using a 10x objective and maximal interval of 22 minutes. After acquisition, time-lapse images were background subtracted (radius 50 pixel), using $\mathrm{FIJ}^{54}$ image analysis software. To analyze cell proliferation and movement within MG overlayed cell cultures, we used an ImageJ plugin, TrackMate ${ }^{55}$, to perform single-particle tracking (SPT). TrackMate offers multiple modes of analysis and based on our cell size and the relatively slow movement of cells, we selected and applied the following parameters in TrackMate: LoG (Laplacian of Gaussian) detector, spot diameter of 35 pixels, threshold 10, median filter and LAP tracker with 15-pixel frame linking, 2- 
frame gap distance and track-segment splitting. We extracted the "spot" count in the desired time frames (one count every 12 hours or 33 frames) to obtain the number of cells or "spots" in the region of interest at those particular time points. We then divided the number of cells in each time point by the number of cells in the starting time point (time $=0$ ) to normalize and plot it as fold increase. A non-parametric t-test using Welch's correction was used to assess significance in fold increase in cell number between the first and the last time point. Plotting and statistical analysis was computed using GraphPad Prism [version 9.1.0 for Windows, GraphPad Software, San Diego, California USA, www.graphpad.com]. To quantify track displacement, we calibrated the images by converting output in pixels into microns, and frames into hours. For visualization of local tracks, we used 50-frame-depth-, time-color-coded tracks overlayed on an GFP or RFPchannel time lapse. For trajectory classification we used the TraJClassifier plugin ${ }^{56}$. The plugin uses TrackMate output data and trajectories and places them into four categories of diffusion/motion, based on mean-square displacement: Normal, Directed, Anomalous (or subdiffusional), and constricted. After all the trajectories were classified into these four categories, we plotted the data using Prism GraphPad Prism 9.1.0. Trajectories from multiple fields of view were plotted (100\% represents all trajectories from one field of view; on average 400-1200 trajectories) and directed-motion trajectories were represented as a percentage of total trajectories. Each dot in a dot plot represents one field of view with the horizontal line depicting the mean of all fields of view per condition. Statistical analysis was computed using Welch's t-test.

\section{Cryo-SEM Sample Preparation and Imaging}

All samples for cryo-SEM were prepared by using a Leica EM HPM100 high pressure freezing (HPF) system. The HPF planchettes were washed by ethanol and exposed to an oxygen plasma for ten minutes prior to use. The frozen hydrated samples were stored in liquid nitrogen. A Leica VCT-100 system was used for subsequent cryotransfer and cryo-imaging. Samples were transferred and coated with sputtered $\mathrm{Au}(2.5 \mathrm{~nm})$ under cryogenic conditions $\left(\mathrm{T}<-135^{\circ} \mathrm{C}\right)$ by using a Leica EM MED020 system. Prior to SEM imaging, sublimation was used to create 
topographic contrast by slightly warming frozen-hydrated samples. SEM imaging were done by using a Zeiss Auriga Cross-Beam FIB-SEM equipped with a Schottky field-emission electron gun (FEG) and an Oxford Max-80 ultrathin window (UTW) silicon-drift detector (SDD) interfaced to an Oxford INCA EDS system. Secondary electron imaging (Everhart-Thornley detector) was done by using $2 \mathrm{keV}$ electrons and $2.5 \mathrm{~nm}$ Au coating.

\section{Assessment of ECM network in MG with OCT}

A spectral domain OCT system with a central wavelength of $\sim 850 \mathrm{~mm}$ was used for MG imaging. The system provides an axial resolution of $\sim 9 \mu \mathrm{m}$ in biological samples (1.4 refractive index assumed) and a transverse resolution of $\sim 5 \mu \mathrm{m}$. Parameters for imaging of $2 \%$ and $25 \%$ MG samples were kept the same. Data processing focused on characterization of the spatial frequency over depth, which is like a previously developed method ${ }^{57}$. Briefly, with the $\mathrm{dB}$ intensity A-scan and the identified sample surface, the intensity profile over $0.4 \mathrm{~mm}$ starting at $\sim 26 \mu \mathrm{m}$ below the sample surface was utilized for analysis. Through the fast Fourier transform, the amplitude spectrum of the spatial frequency was obtained, and the exponential fit of the local peaks was performed. The exponential decay coefficient was extracted as the measure of the spatial frequency over depth and was used for comparison between the $2 \%$ and $25 \%$ MG samples. A higher decay coefficient represents relatively stronger low-frequency components of the spatial frequency spectrum.

\section{Rotational Rheology}

The linear viscoelastic material functions, the storage modulus, $G^{\prime}$, and the loss modulus, $G^{\prime \prime}$, of the $2 \%, 25 \%$ MG samples, as well as ascites fluids isolated from ovarian cancer patients were characterized using an Advanced Rheometric Expansion System (ARES) rheometer available from TA Instruments of New Castle, DE. The rotational rheometer was used with stainless steel parallel disks with $25 \mathrm{~mm}$ diameters and had a force rebalance transducer $0.2 \mathrm{~K}-\mathrm{FRTN} 1$. The actuator of the ARES is a DC servomotor with a shaft supported by an air bearing with an angular displacement range of $5 \times 10^{-6}$ to $0.5 \mathrm{rad}$, and angular frequency range of $1 \times 10^{-5}$ to $100 \mathrm{rad} / \mathrm{s}$. The 
angular velocity range is $1 \times 10^{-6}$ to $200 \mathrm{rad} / \mathrm{s}$. The sample loading procedure was the same for all the experiments and the gap height between two disks was kept constant at $1 \mathrm{~mm}$. Sufficient volume of samples were used to fill the gap between parallel disks, and the linear viscoelastic properties of the samples were collected as a function of frequency at constant strain of $50 \%$ and room temperature. The samples were not pre-sheared. For discussion of dynamic property characterization of complex fluids see, for example, Bird, R.B., Armstrong, R.C. and Hassager, O. “Dynamics of polymeric liquids. Wiley, 1987. “

Author Contribution: S.A., T.P. and M.I. design and conceptualization the stud with significant input from S.F and P.B. D.C., and D.K. IHC analysis of human tumors and PDX tissue sections was performed by S.A. T.P., perform cell tracking experiments and image quantification. S.F. performed mesothelial clearance assays. P.B., L.Q., D.Kh. designed and performed shear-stress rheometric analyses. P.B. and W.L. designed and performed electron microscopy experiments. M.R., P.J., E.D., E.N-M., and M.P-Z., identified human tumor samples and performed pathologic analysis of laminin $\gamma 1$ expression in tumors representing disease before and after chemotherapy. S.W. designed and performed optical coherence tomography experiments and contributed to writing the manuscript. S.A., T.P., and M.I., wrote a manuscript with significant input (methodology description) from S.F., P.B., L.Q., D.Kh., S.W., and M.P-Z.

Acknowledgments. We would like to thank the Stevens Institute of Technology, Multiscale Imaging Center led by Dr. Matthew Libera. We are especially grateful for technical help with sample preparation and processing that was provided by Dr. Tsengming Chou. We thank Thomas Cattabiani for editorial help with the manuscript preparation. This study was supported by NIH/NCI R21CA256615 (M.I. and S.W.) and Kaleidoscope of Hope Ovarian Cancer Foundation (M. I). 
Competing Interest Statement. The authors declare no competing interests

\section{Figure Legends}

Figure 1. IHC evaluation of laminin $\gamma 1$ and collagens in PAX8-positive OC outgrowths and PAX8-negative surrounding connective tissue. $(\boldsymbol{A}, \boldsymbol{E})$ Representative images of laminin $\gamma 1$, and $(\boldsymbol{C}, \boldsymbol{G})$ collagen expression in OC outgrowths and extra-tumoral connective tissue protruding from the surface of the human ovary $(\mathbf{A}, \mathbf{C})$, or in xenograft experiments, from the surface of the omentum (E, G). Red arrows point to the deposition of laminin $\gamma 1$. Green arrows indicate fibrillar collagen structures in a tumor's connective tissue, whereas black arrows represent collagen deposition within tumors. $(\boldsymbol{B}, \boldsymbol{F})$ Quantification of laminin $\gamma 1$ and $(\boldsymbol{D}, \boldsymbol{H})$ collagen expression in PAX8-positive tumor outgrowths and PAX8-negative surrounding tissue. Each data point represents one region of interest within a tumor or surrounding stroma tissue, and the line reflects the median of the data point distribution. An unpaired, two-tailed, non-parametric student t-test was used to examine statistical differences between data points distributions representing tumor and extra-tumoral stroma. ${ }^{*}-\mathrm{p}<0.05$. Bars are $100 \mu \mathrm{m}$. "No tissue" values represent background level after RGB image deconvolution and image conversion to 8-bit gray scale (0-255 pixels range $)^{53}$

Figure 2. OC before and after chemotherapy is associated with spatially distinct laminin y1 and collagen ECM microenvironments. (A) Hematoxylin and PAX8 stain of high-grade serous ovarian cancer (HGSOC) growing within the ovary before chemotherapy; bar is $100 \mu \mathrm{m}$. (B) Laminin Y1 and (C) collagens deposition in matched omental tissue of HGSOC before and after taxane-platin based treatment; bar is $500 \mu \mathrm{m}$. (D) $\mathrm{H}$-score based quantification of laminin $\mathrm{y} 1$ positivity in matched tissue samples representing HGSOC before and after taxane-platin based 
therapy. Tissues were analyzed from three patients. Wilcoxon sum rank test was used to compare the difference between the groups, NS= Not-Statistically different. (E) Quantification of collagens expression in omental metastases before and after taxane-platin based chemotherapy. An unpaired, two-tailed, non-parametric student t-test was used to examine statistical differences between data points.

\section{Figure 3. Laminin and collagen-rich ECM reconstitution stimulates outgrowths in} suspended cultures of various ovarian cancer cells. (A) Graphical representation of the assay design to study outgrowth dynamics. (B) Representative bright-field images of outgrowth formation in FNE cells expressing plasmid containing m-p53 ${ }^{\mathrm{R} 175 \mathrm{H}}$ (FNE-m-p53), or control plasmid, and cultured as suspended clusters reconstituted with $2 \% \mathrm{MG}$. Cell structures were cultured for 7 days before imaging and subsequently flowed for additional 72 hrs. (C)

Quantification of 3D structure expansion, each dot represents fold change in area, over 72 hrs of filming time, in one structure. (D) Quantification of outgrowths in FNE m-p53 or FNE control cells. Bar represents and average percentage outgrowth formation in three experiment with at least 30 suspended cell clusters analyzed per experiment. (E) Representative bright-field images of outgrowths in Hey-A8 and Tyk-nu OC cell lines, and quantification of outgrowths in Hey-A8 or Tyk-nu. Bars represent an average percentage of outgrowths formation in three independent experiments with 20-30 structures scored per experiment. Scale bars are $200 \mu \mathrm{m}$.

Figure 4. Detached outgrowths clear the mesothelial monolayers. (A) Schematic representation of Hey-A8 outgrowth detachment and co-culture with mesothelial cells expressing GFP. (B) Representative bright-field and corresponding fluorescent images of mesothelial clearance by Hey-A8 cell cluster (black arrow) or detached outgrowths (white arrows); bar is 200 $\mu m$. (C) Quantification of mesothelial clearance by Hey-A8 cell clusters and detached outgrowths. Each data point represents a cell cluster or outgrowth. Twenty outgrowths and cell clusters 
representing two independent experiments were analyzed. * $p<0.05$, for an unpaired, nonparametric student t-test. (D) Representative bright-field and corresponding Z fluorescent maximum projection images of Hey-A8 cell clusters forming outgrowths and treated with propidium iodide $(\mathrm{PI})$ to spatially visualize cell death within the structure. (E) Quantification of PI incorporation by cell clusters or outgrowths. Sixteen Hey-A8 cell structures were analyzed in two independent experiments.

Figure 5. Elevation of ECM content inhibits outgrowths. (A) Representative bright-field maximum Z-projection images of FNE-m-p53. Bar is $250 \mu m$. (B) Hey-A8 cells cultured under suspended conditions and reconstituted with $2 \%$ or $25 \%$ MG, bar is $500 \mu \mathrm{m}$. Boxed area shows cell cluster structure edge. Dot plots $(A, B)$ represent distribution of outgrowth lengths from individual cell clusters reconstituted with $2 \%$ or $25 \%$ MG. * denotes $p<0.05$, for an unpaired, nonparametric student-t test (C) Representative electron scanning micrographs representing $2 \%$ and $25 \%$ MG. (D) Representative OCT B-scan images of 2\% and 25\% MG. Scale bars are $100 \mu \mathrm{m}$.

(E) Representative OCT depth-resolved intensity profiles for spatial frequency analysis. (E) Amplitude spectra of the spatial frequency corresponding to (F) showing relatively stronger lowfrequency components from $25 \%$ MG, and exponential fit of local peaks indicating a faster decay rate in $25 \%$ MG.

Figure 6 ECM modulates viscoelastic properties in the OC culture microenvironment. (A, B) Measurements of storage (elastic) and loss (viscous) moduli in OC cell culture media reconstituted with $2 \%$ or $25 \%$ of MG. (C,D) Graphs represent calculated tan $\delta$ from values reported in A, B. or in ascitic fluids isolated from OC patients with relapsed disease $(E) .(C, D, F)$ and $E .(G, H, I)$ Scanning electron micrographs representing ascites fibrous network surrounding tumor cells. 
Figure 7. Elevation of ECM levels suppress cell translocation. (A) Representative phase contrast-, fluorescent- and cell-trajectory images of cell translocation within FNE-m-p53 cell monolayers overlayed with $2 \%$ or (B) $25 \% \mathrm{M}$. (C) Representative images of cell trajectories dynamics. (D) Distribution of \% number of classified cell trajectories within FNE-m-p53 cell monolayers overlayed with various concentrations of MG. 400-1000 cell trajectories were quantified within multiple fields of view representing each condition. $p$ value was derived using Welch's t-test. (E) Graphical representation of key findings in this study.

\section{S. Figure 1. Elevation of ECM content suppresses FNE-m-p53 and Hey-A8 cell proliferation.}

Quantification of an area in (A) FNE-m-p53 or (B) Hey-8A suspended cell clusters reconstituted with $2 \%$ or $25 \%$ MG. Dots represent individual cell cluster structure measured 9 days after starting the culture. (C) Cartoon representation of the experimental design for ECM encapsulation of cell monolayers. Representative fluorescent images of (D) FNE-m-p53 or (E) Hey-A8 cells expressing RFP grown under condition of $2 \%$ or $25 \%$ MG encapsulation. Dot plots represent fold change increase in cell number over time. Each dot corresponds to one field of view. (F) Representative fluorescent images of EdU and Hoechst in suspended Hey-A8 cells and reconstituted with $2 \%$ and $25 \%$ MG. Cell structures were processed 9 days after starting the culture. Dot plot represents the fraction of EdU positive cells. Each dot corresponds to one structure. Welch's t-test was used to compute statistical difference. (G) Phase contrast images of FNE-m-p53 and Hey-A8 cells grown as 2D adherent cultures. Scale bars are 100 $\mathrm{mm}$. 


\section{Supplementary Movies.}

MOVIE 1. Outgrowth formation by FNE-m-p53 suspended cellular clusters reconstituted with $2 \%$ MG. Cell clusters were imaged for 5 days with 2 hrs intervals.

MOVIE 2. Outgrowth formation by Hey-8A suspended cellular clusters reconstituted with $2 \%$ MG. Cell clusters were imaged for 5 days with 2 hrs intervals.

MOVIE 3. Outgrowth formation by Tyk-nu suspended cellular clusters reconstituted with $2 \%$ MG. Cell clusters were imaged for 5 days with 2 hrs intervals.

MOVIE 4. FNE-m-p53-GFP cell proliferation in 2\% and 25\% MG. Images were obtained every 22 minutes for a duration of $61 \mathrm{hrs}$.

MOVIE 5. Hey-A8-mKO2 cell proliferation in 2\% and 25\% MG. Images were obtained every 22 minutes for a duration of $68 \mathrm{hrs}$.

MOVIE 6. Evolution of cell movement trajectories in FNE-m-p53 monolayer cultures encapsulated with $2 \%$ MG. Images were obtained every 22 minutes for a duration of $61 \mathrm{hrs}$.

MOVIE 7. Evolution of cell movement trajectories in FNE-m-p53 monolayer cultures encapsulated with $25 \%$ MG. Images were obtained every 22 minutes for a duration of $68 \mathrm{hrs}$. 


\section{References}

$1 \quad$ Kurman, R. J. \& Shih le, M. The origin and pathogenesis of epithelial ovarian cancer: a proposed unifying theory. Am J Surg Pathol 34, 433-443, doi:10.1097/PAS.0b013e3181cf3d79 (2010).

2 Auersperg, N. Ovarian surface epithelium as a source of ovarian cancers: unwarranted speculation or evidence-based hypothesis? Gynecol Oncol 130, 246-251, doi:10.1016/j.ygyno.2013.03.021 (2013).

3 Folkins, A. K. et al. A candidate precursor to pelvic serous cancer (p53 signature) and its prevalence in ovaries and fallopian tubes from women with BRCA mutations. Gynecol Oncol 109, 168-173, doi:S0090-8258(08)00007-3 [pii]

10.1016/j.ygyno.2008.01.012 (2008).

4 Meserve, E. E. K., Brouwer, J. \& Crum, C. P. Serous tubal intraepithelial neoplasia: the concept and its application. Mod Pathol 30, 710-721, doi:10.1038/modpathol.2016.238 (2017).

5 Karst, A. M. \& Drapkin, R. Ovarian cancer pathogenesis: a model in evolution. J Oncol 2010, 932371, doi:10.1155/2010/932371.

6 Lengyel, E. Ovarian cancer development and metastasis. Am J Pathol 177, 1053-1064, doi:10.2353/ajpath.2010.100105 (2010).

7 Ahmed, N. \& Stenvers, K. L. Getting to know ovarian cancer ascites: opportunities for targeted therapy-based translational research. Front Oncol 3, 256, doi:10.3389/fonc.2013.00256 (2013).

$8 \mathrm{Li}, \mathrm{X}$. et al. N-linked glycosylation on laminin gamma1 influences recognition of antilaminin gamma1 pemphigoid autoantibodies. J Dermatol Sci 77, 125-129, doi:10.1016/j.jdermsci.2014.12.003 (2015).

9 Hennet, T. Collagen glycosylation. Curr Opin Struct Biol 56, 131-138, doi:10.1016/j.sbi.2019.01.015 (2019).

10 Chen, D. et al. Differential expression of collagen- and laminin-binding integrins mediates ureteric bud and inner medullary collecting duct cell tubulogenesis. American journal of physiology. Renal physiology 287, F602-611, doi:10.1152/ajprenal.00015.2004 (2004).

11 Chen, C. S., Tan, J. \& Tien, J. Mechanotransduction at cell-matrix and cell-cell contacts. Annu Rev Biomed Eng 6, 275-302, doi:10.1146/annurev.bioeng.6.040803.140040 (2004).

12 Orr, A. W., Helmke, B. P., Blackman, B. R. \& Schwartz, M. A. Mechanisms of mechanotransduction. Dev Cell 10, 11-20, doi:S1534-5807(05)00482-X [pii]

10.1016/j.devcel.2005.12.006 (2006).

13 Wang, C. et al. Type III collagen is a key regulator of the collagen fibrillar structure and biomechanics of articular cartilage and meniscus. Matrix Biol 85-86, 47-67, doi:10.1016/j.matbio.2019.10.001 (2020).

14 Spofford, C. M. \& Chilian, W. M. Mechanotransduction via the elastin-laminin receptor (ELR) in resistance arteries. J Biomech 36, 645-652, doi:10.1016/s0021-9290(02)004426 (2003).

15 Banno, A. \& Ginsberg, M. H. Integrin activation. Biochem Soc Trans 36, 229-234, doi:BST0360229 [pii]

10.1042/BST0360229 (2008).

16 Schwartz, M. A. \& Shattil, S. J. Signaling networks linking integrins and rho family GTPases. Trends Biochem Sci 25, 388-391, doi:S0968-0004(00)01605-4 [pii] (2000).

17 Fields, B. et al. The Adult Murine Intestine is Dependent on Constitutive Laminin-gamma1 Synthesis. Sci Rep 9, 19303, doi:10.1038/s41598-019-55844-x (2019).

18 Willem, M. et al. Specific ablation of the nidogen-binding site in the laminin gamma1 chain interferes with kidney and lung development. Development 129, 2711-2722 (2002). 
19 Jailkhani, N. et al. Noninvasive imaging of tumor progression, metastasis, and fibrosis using a nanobody targeting the extracellular matrix. Proc Natl Acad Sci U S A 116, 1418114190, doi:10.1073/pnas.1817442116 (2019).

20 Winkler, J., Abisoye-Ogunniyan, A., Metcalf, K. J. \& Werb, Z. Concepts of extracellular matrix remodelling in tumour progression and metastasis. Nature communications 11 , 5120, doi:10.1038/s41467-020-18794-x (2020).

21 Kuhn, E. et al. The diagnostic and biological implications of laminin expression in serous tubal intraepithelial carcinoma. Am J Surg Pathol 36, 1826-1834, doi:10.1097/PAS.0b013e31825ec07a (2012).

22 Iwanicki, M. P. et al. Mutant p53 regulates ovarian cancer transformed phenotypes through autocrine matrix deposition. JCI Insight 1, doi:10.1172/jci.insight.86829 (2016).

23 Rentchler, E. C., Gant, K. L., Drapkin, R., Patankar, M. \& P, J. C. Imaging Collagen Alterations in STICs and High Grade Ovarian Cancers in the Fallopian Tubes by Second Harmonic Generation Microscopy. Cancers (Basel) 11, doi:10.3390/cancers11111805 (2019).

24 Benton, G., Arnaoutova, I., George, J., Kleinman, H. K. \& Koblinski, J. Matrigel: from discovery and ECM mimicry to assays and models for cancer research. Adv Drug Deliv Rev 79-80, 3-18, doi:10.1016/j.addr.2014.06.005 (2014).

25 Mroue, R. \& Bissell, M. J. Three-dimensional cultures of mouse mammary epithelial cells. Methods Mol Biol 945, 221-250, doi:10.1007/978-1-62703-125-7_14 (2013).

26 Farsinejad, S., Cattabiani, T., Muranen, T. \& Iwanicki, M. Ovarian Cancer DisseminationA Cell Biologist's Perspective. Cancers (Basel) 11, doi:10.3390/cancers11121957 (2019).

27 Muranen, T. et al. Inhibition of PI3K/mTOR leads to adaptive resistance in matrix-attached cancer cells. Cancer Cell 21, 227-239, doi:10.1016/j.ccr.2011.12.024 (2012).

28 Zhang, S. et al. Both fallopian tube and ovarian surface epithelium are cells-of-origin for high-grade serous ovarian carcinoma. Nature communications 10, 5367, doi:10.1038/s41467-019-13116-2 (2019).

29 Zhang, S. et al. Genetically Defined, Syngeneic Organoid Platform for Developing Combination Therapies for Ovarian Cancer. Cancer discovery 11, 362-383, doi:10.1158/2159-8290.CD-20-0455 (2021).

30 Hill, S. J. et al. Prediction of DNA Repair Inhibitor Response in Short-Term Patient-Derived Ovarian Cancer Organoids. Cancer Discov 8, 1404-1421, doi:10.1158/2159-8290.CD-180474 (2018).

31 Hoffmann, K. et al. Stable expansion of high-grade serous ovarian cancer organoids requires a low-Wnt environment. EMBO J, e104013, doi:10.15252/embj.2019104013 (2020).

32 Wegner, K. A., Keikhosravi, A., Eliceiri, K. W. \& Vezina, C. M. Fluorescence of Picrosirius Red Multiplexed With Immunohistochemistry for the Quantitative Assessment of Collagen in Tissue Sections. J Histochem Cytochem 65, 479-490, doi:10.1369/0022155417718541 (2017).

33 Kleinman, $\mathrm{H}$. K. et al. Basement membrane complexes with biological activity. Biochemistry 25, 312-318, doi:10.1021/bi00350a005 (1986).

34 Martin, G. R., Timpl, R. \& Kuhn, K. Basement membrane proteins: molecular structure and function. Adv Protein Chem 39, 1-50 (1988).

35 Aisenbrey, E. A. \& Murphy, W. L. Synthetic alternatives to Matrigel. Nat Rev Mater 5, 539551, doi:10.1038/s41578-020-0199-8 (2020).

36 Domcke, S., Sinha, R., Levine, D. A., Sander, C. \& Schultz, N. Evaluating cell lines as tumour models by comparison of genomic profiles. Nature communications 4, 2126, doi:10.1038/ncomms3126 (2013).

37 Iwanicki, M. P. et al. Ovarian cancer spheroids use myosin-generated force to clear the mesothelium. Cancer discovery 1, 144-157, doi:10.1158/2159-8274.CD-11-0010 (2011). 
38 Davidowitz, R. A., Iwanicki, M. P. \& Brugge, J. S. In vitro mesothelial clearance assay that models the early steps of ovarian cancer metastasis. J Vis Exp, doi:10.3791/3888 (2012).

39 Abugomaa, A. et al. Establishment of 2.5D organoid culture model using 3D bladder cancer organoid culture. Sci Rep 10, 9393, doi:10.1038/s41598-020-66229-w (2020).

40 Barriga, E. H. \& Mayor, R. Adjustable viscoelasticity allows for efficient collective cell migration. Semin Cell Dev Biol 93, 55-68, doi:10.1016/j.semcdb.2018.05.027 (2019).

41 Aumailley, M. The laminin family. Cell Adh Migr 7, 48-55, doi:10.4161/cam.22826 (2013).

42 Yurchenco, P. D. Basement membranes: cell scaffoldings and signaling platforms. Cold Spring Harbor perspectives in biology 3, doi:10.1101/cshperspect.a004911 (2011).

43 Kyprianou, C. et al. Basement membrane remodelling regulates mouse embryogenesis. Nature 582, 253-258, doi:10.1038/s41586-020-2264-2 (2020).

44 Delaine-Smith, R. M. et al. Modelling TGFbetaR and Hh pathway regulation of prognostic matrisome molecules in ovarian cancer. iScience 24, 102674, doi:10.1016/j.isci.2021.102674 (2021).

45 Raspanti, M., Viola, M., Sonaggere, M., Tira, M. E. \& Tenni, R. Collagen fibril structure is affected by collagen concentration and decorin. Biomacromolecules 8, 2087-2091, doi:10.1021/bm070091t (2007).

46 Plou, J. et al. From individual to collective 3D cancer dissemination: roles of collagen concentration and TGF-beta. Sci Rep 8, 12723, doi:10.1038/s41598-018-30683-4 (2018).

47 Palecek, S. P., Loftus, J. C., Ginsberg, M. H., Lauffenburger, D. A. \& Horwitz, A. F. Integrin-ligand binding properties govern cell migration speed through cell-substratum adhesiveness. Nature 385, 537-540, doi:10.1038/385537a0 (1997).

48 Webb, D. J., Parsons, J. T. \& Horwitz, A. F. Adhesion assembly, disassembly and turnover in migrating cells -- over and over and over again. Nat Cell Biol 4, E97-100, doi:10.1038/ncb0402-e97

ncb0402-e97 [pii] (2002).

49 Chaudhuri, O., Cooper-White, J., Janmey, P. A., Mooney, D. J. \& Shenoy, V. B. Effects of extracellular matrix viscoelasticity on cellular behaviour. Nature 584, 535-546, doi:10.1038/s41586-020-2612-2 (2020).

50 Chaudhuri, O. et al. Extracellular matrix stiffness and composition jointly regulate the induction of malignant phenotypes in mammary epithelium. Nature materials 13, 970-978, doi:10.1038/nmat4009 (2014).

51 Byers, L. J. et al. Increased levels of laminin in ascitic fluid of patients with ovarian cancer. Cancer Lett 88, 67-72, doi:10.1016/0304-3835(94)03625-s (1995).

52 Uphoff, C. C. \& Drexler, H. G. Detection of mycoplasma contaminations. Methods Mol Biol 290, 13-23, doi:10.1385/1-59259-838-2:013 (2005).

53 Lee, Y. Y. et al. Type 1 Diabetes Induces Hearing Loss: Functional and Histological Findings in An Akita Mouse Model. Biomedicines 8, doi:10.3390/biomedicines8090343 (2020).

54 Schindelin, J. et al. Fiji: an open-source platform for biological-image analysis. Nat Methods 9, 676-682, doi:10.1038/nmeth.2019 (2012).

55 Tinevez, J. Y. et al. TrackMate: An open and extensible platform for single-particle tracking. Methods 115, 80-90, doi:10.1016/j.ymeth.2016.09.016 (2017).

56 Wagner, T., Kroll, A., Haramagatti, C. R., Lipinski, H. G. \& Wiemann, M. Classification and Segmentation of Nanoparticle Diffusion Trajectories in Cellular Micro Environments. PLoS One 12, e0170165, doi:10.1371/journal.pone.0170165 (2017).

57 Wang, S. et al. Three-dimensional computational analysis of optical coherence tomography images for the detection of soft tissue sarcomas. J Biomed Opt 19, 21102, doi:10.1117/1.JBO.19.2.021102 (2014). 


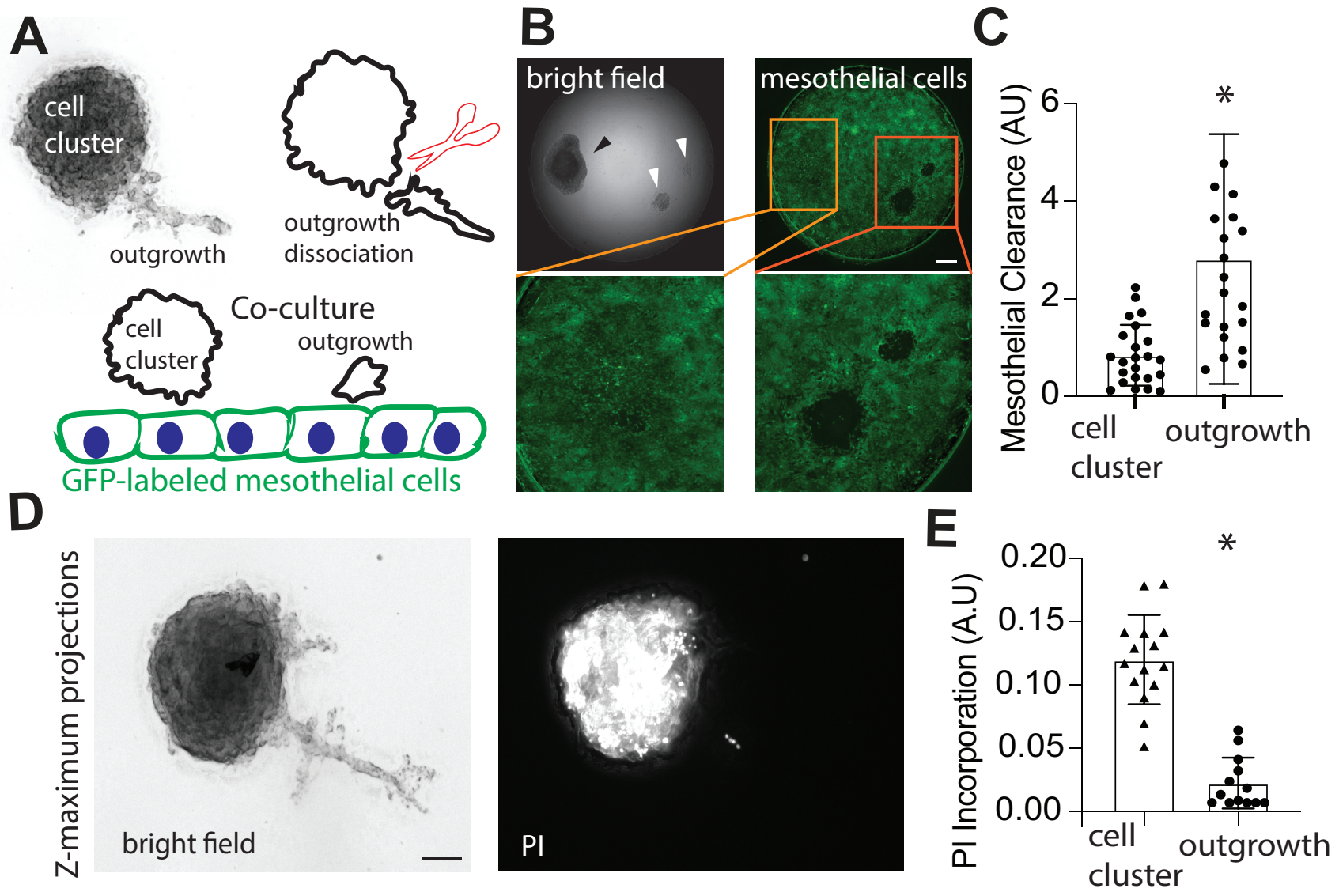

Figure 4. Detached outgrowths clear the mesothelial monolayers. (A) Schematic representation of Hey-A8 outgrowth detachment and co-culture with mesothelial cells expressing GFP. Representative bright-field and corresponding fluorescent images of mesothelial clearance by Hey-A8 cell cluster (black arrow) or detached outgrowths (white arrows); bar is $200 \mu \mathrm{m}$. (C) Quantification of mesothelial clearance by Hey-A8 cell clusters and detached outgrowths. Each data point represents a cell cluster or outgrowth. Twenty outgrowths and cell clusters representing two independent experiments were analyzed. ${ }^{*} \mathrm{p}<0.05$, for an unpaired, non-parametric student $\mathrm{t}$-test. (D) Representative bright-field and corresponding fluorescent maximum projection images of Hey-A8 cell clusters forming outgrowth and treated with propidium iodide (PI) to spatially visualize cell death within the structure. (E) Quantification of PI incorporation by cell clusters or outgrowths. Sixteen Hey-A8 cell structures were analyzed in two independent experiments. 

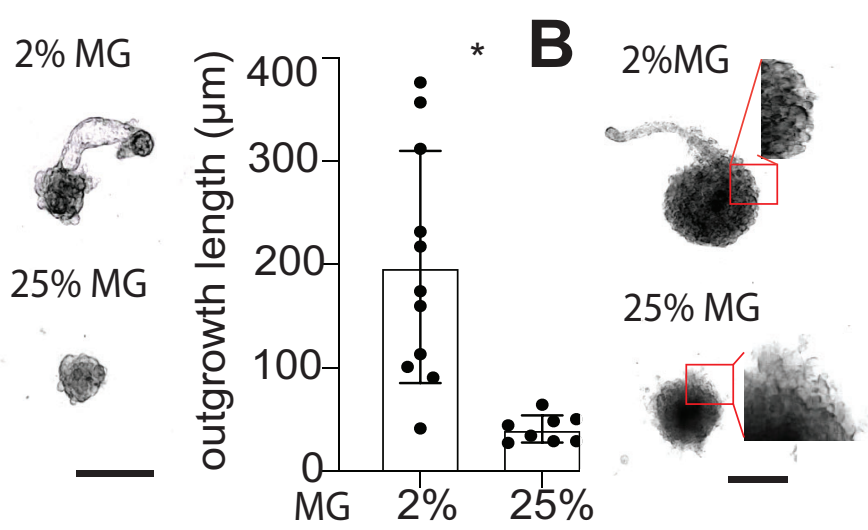

D

E
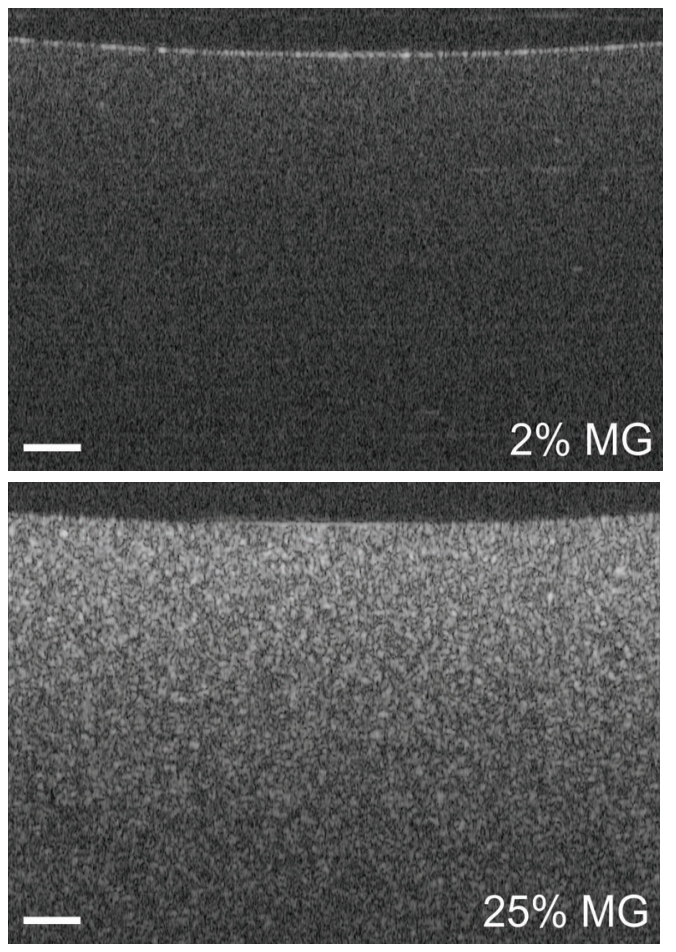
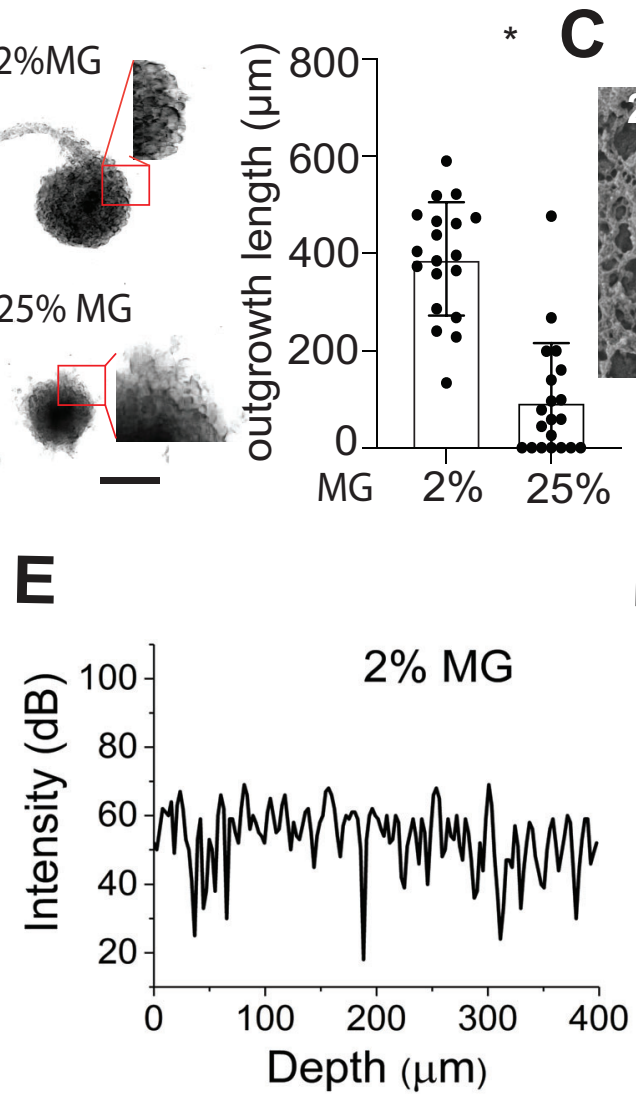

F
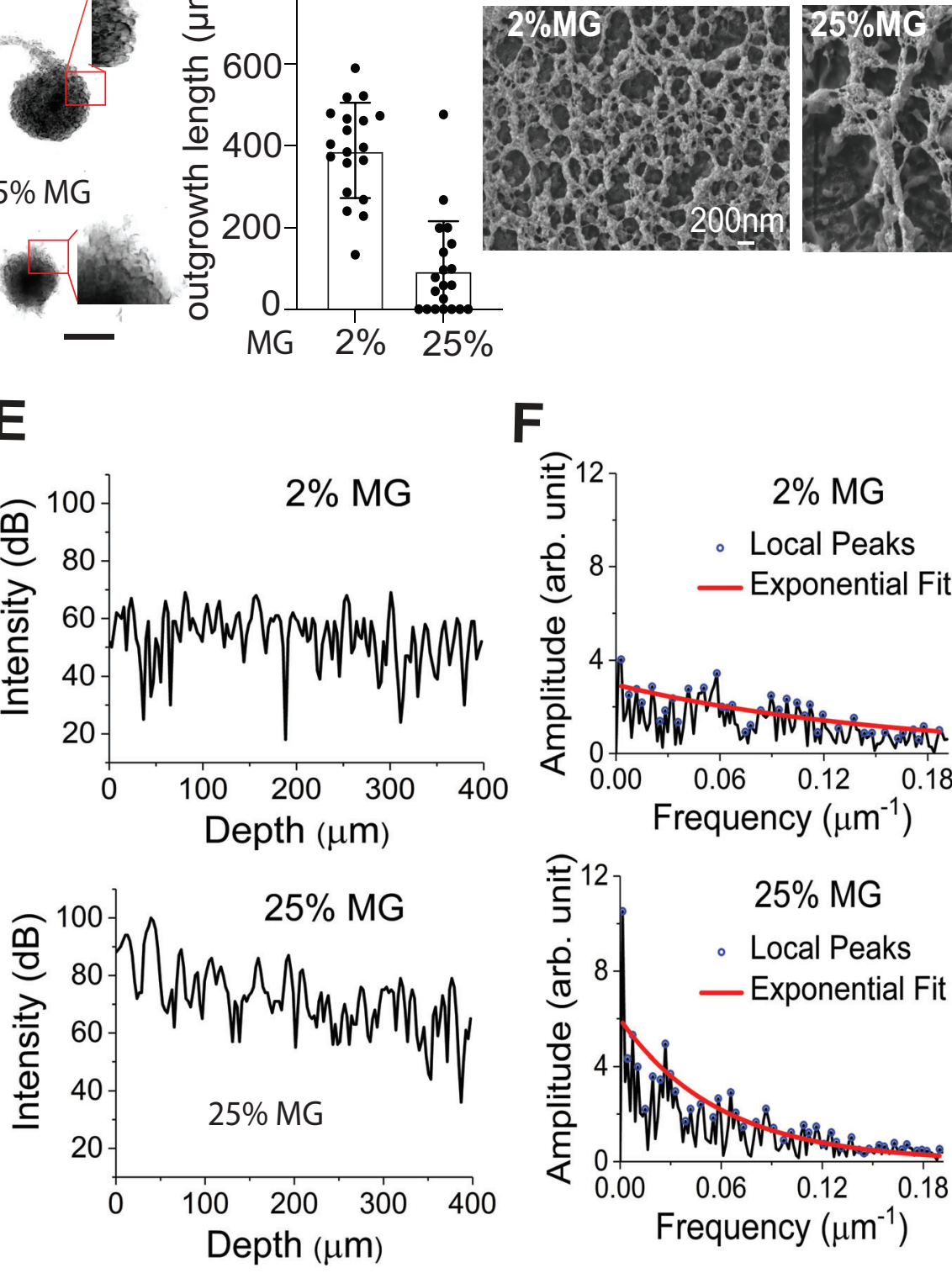

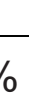
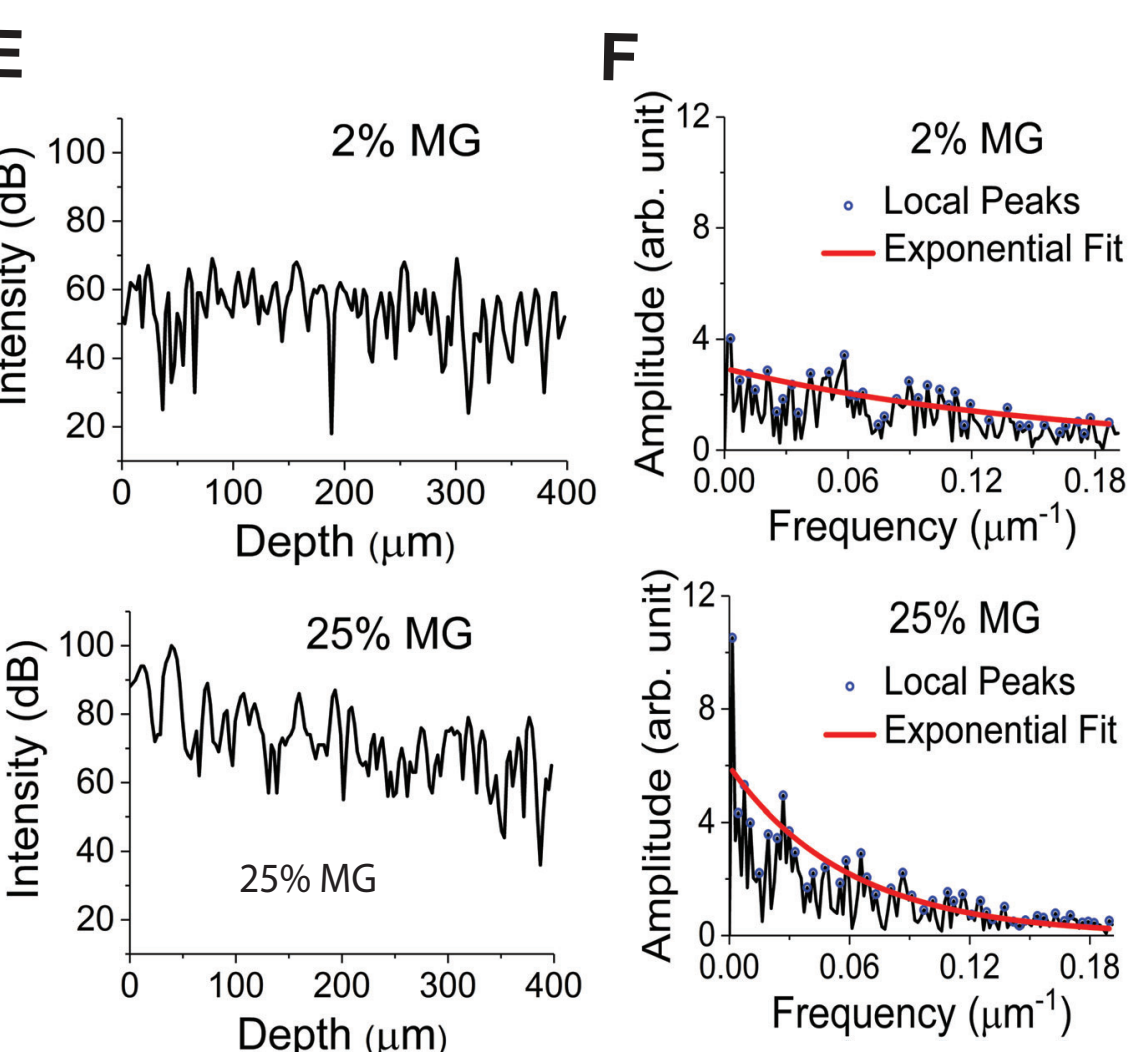

Figure 5. Elevation of ECM levels inhibits outgrowths. (A) Representative bright-field maximum Z-projection images of FNE-m-p53, bar is $250 \mu \mathrm{m}$, or (B) Hey-8A cells cultured under suspended conditions and reconstituted with $2 \%$ or $25 \%$ MG, bar is $500 \mu \mathrm{m}$. Boxed area shows cell cluster structure edge. FNE-m-p53 and Hey-A8 cell clusters were grown for 9 days and the images show the final time point. Dot plots $(A, B)$ represent distribution of outgrowth lengths from individual cell clusters reconstituted with $2 \%$ or $25 \%$ MG. * Denotes $p<0.05$, for Welch's t-test. (C) Representative electron scanning micrography representing $2 \%$ and $25 \%$ MG. (D) Representative OCT B-scan images of 2\% and 25\% MG. Scale bars are $100 \mu \mathrm{m}$. (E) Representative OCT depth-resolved intensity profiles for spatial frequency analysis. (F) Amplitude spectra of the spatial frequency corresponding to $(F)$ showing relatively stronger low-frequency components from $25 \% \mathrm{MG}$, and exponential fit of local peaks indicating a faster decay rate in $25 \%$ MG. 

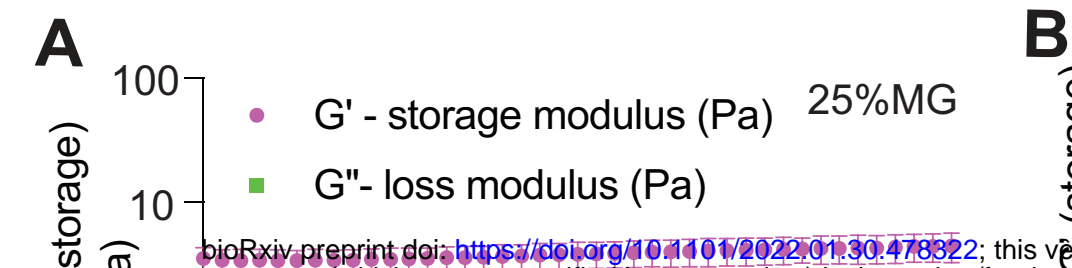

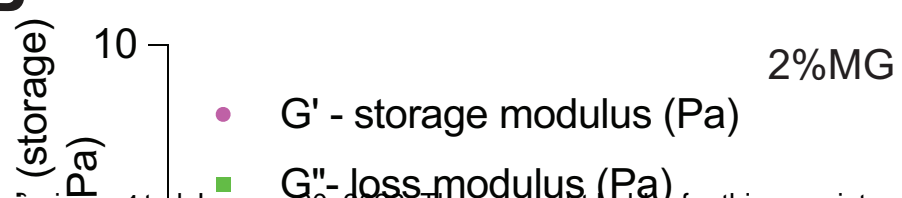

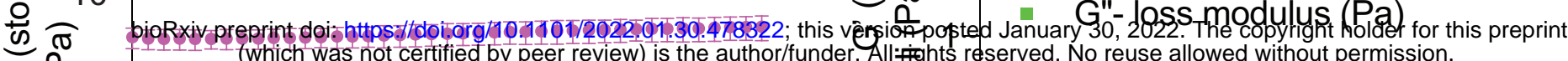
in (which was not certified by peer review) is the author/funder. All:

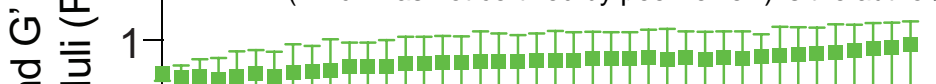

듬

क है $0.1-$

0.01

IIIIIIIIIIIIIIIIIIIIIIIIII

10

C

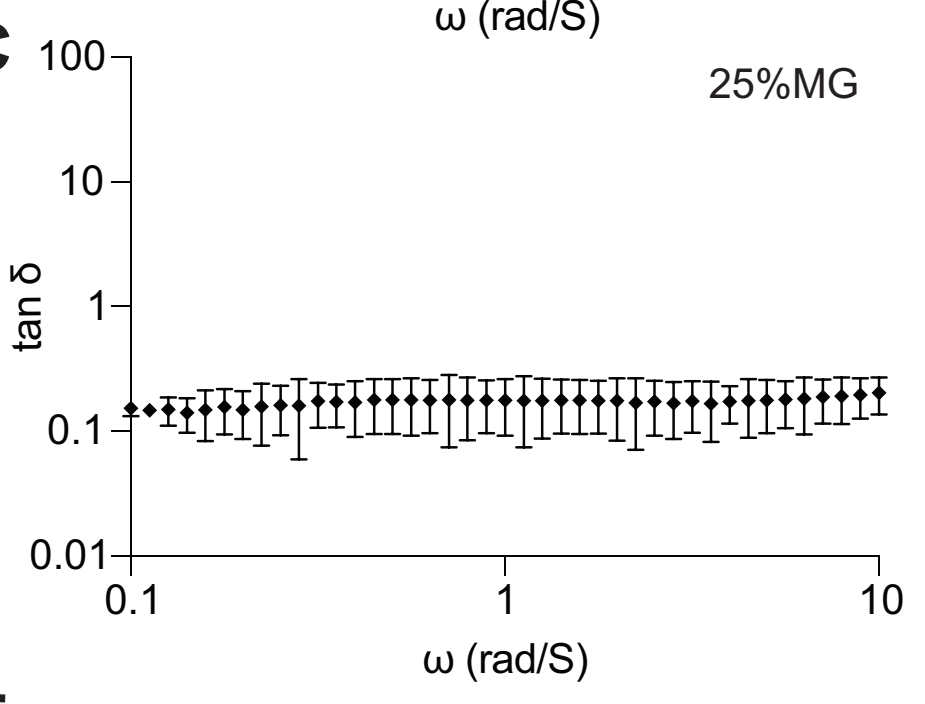

$\mathbf{E}$

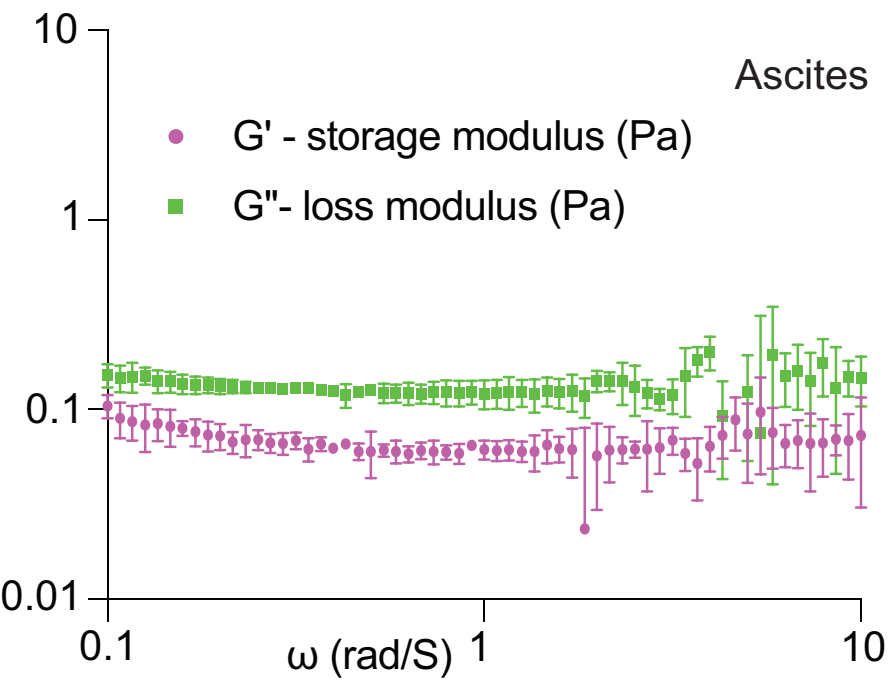

D

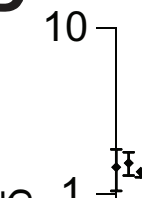

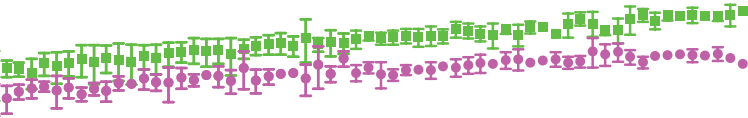
क $\stackrel{0}{=}$

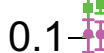
రे

స్

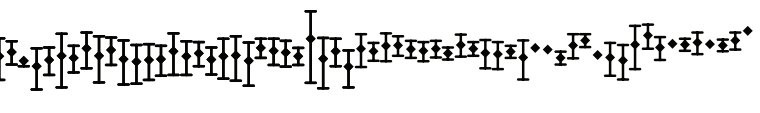

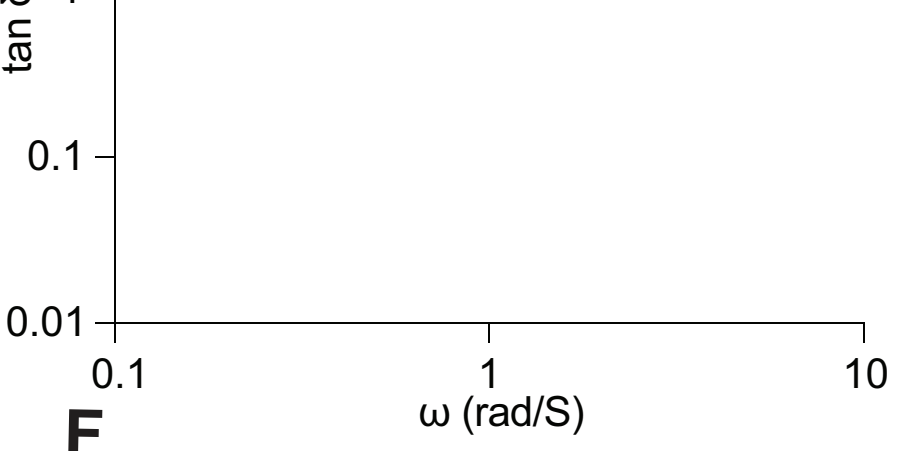

Ascites

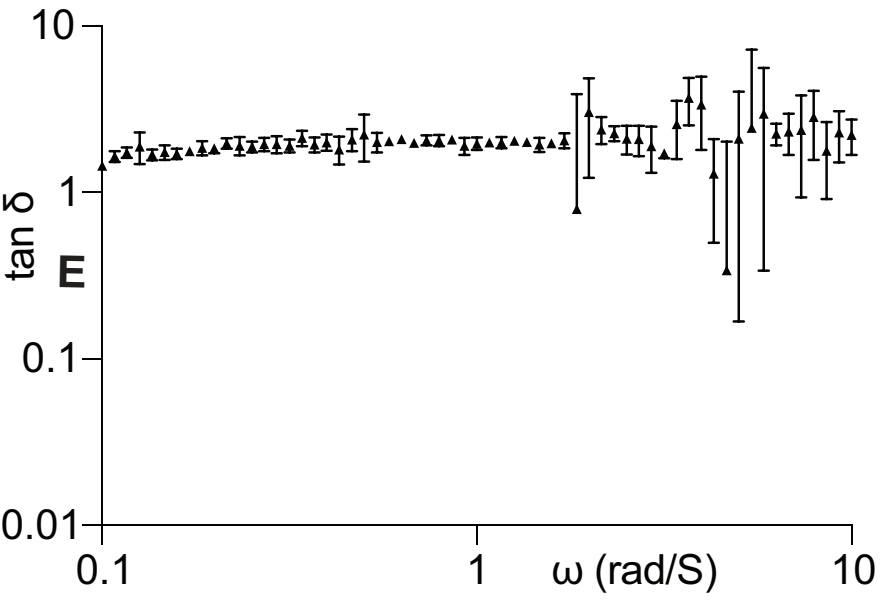

G

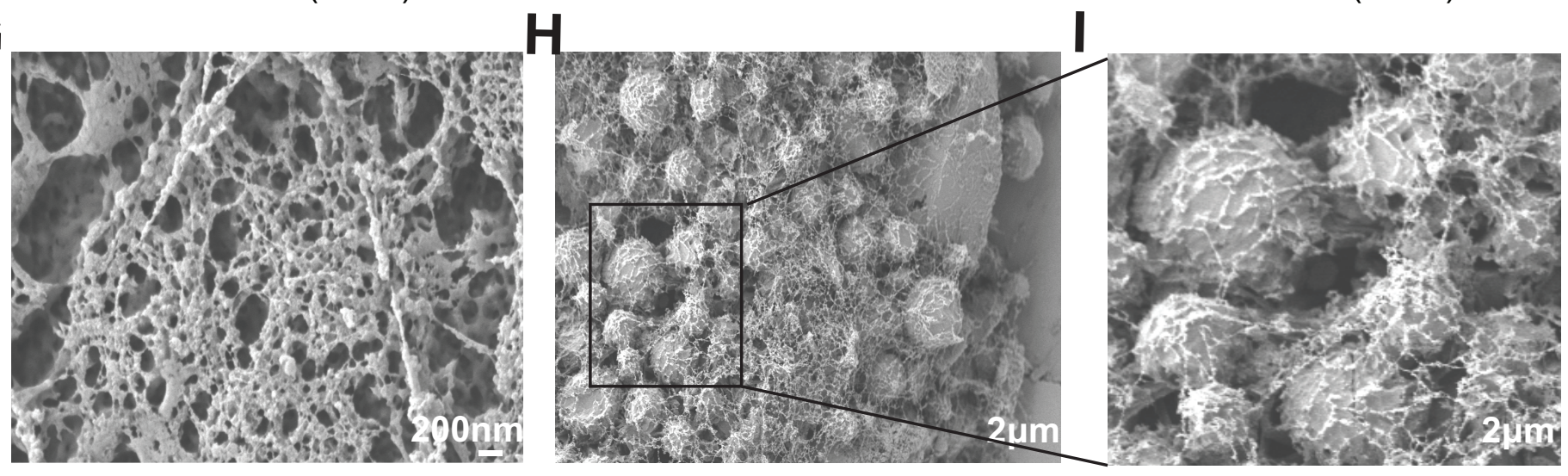

Figure 6. ECM levels modulate viscoelastic properties in the OC culture microenvironment. Measurements of storage (elastic) and loss (viscous) moduli in OC cell culture media reconstituted with $2 \%$ or $25 \%$ of MG (A, B); or in ascitic fluids isolated from OC patients with relapsed disease (E). (C, D, F) Graphs represent calculated tan $\delta$ from values reported in A, B and E. (G, H,I) Scanning electron micrographs representing ascites fibrous ECM network surrounding tumor cells. 
A
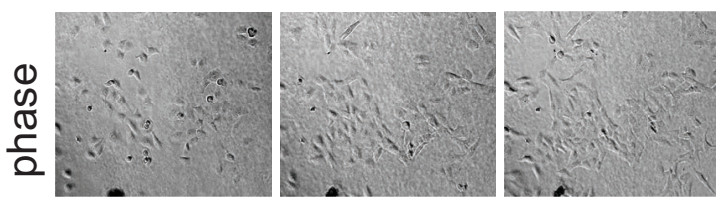

$\frac{0}{\frac{1}{\alpha}}$
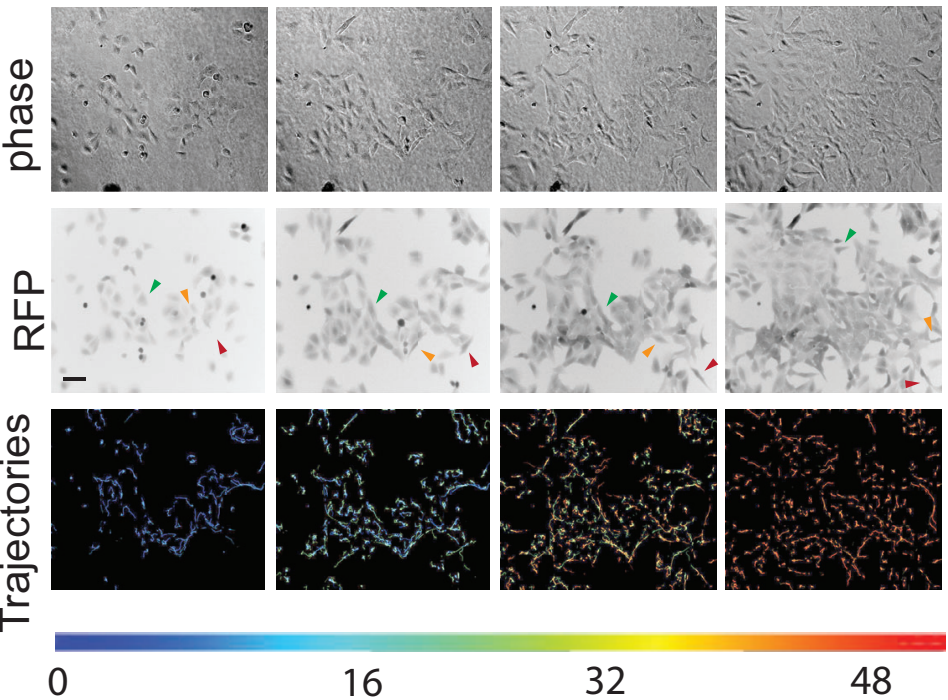

32

Time (hrs)
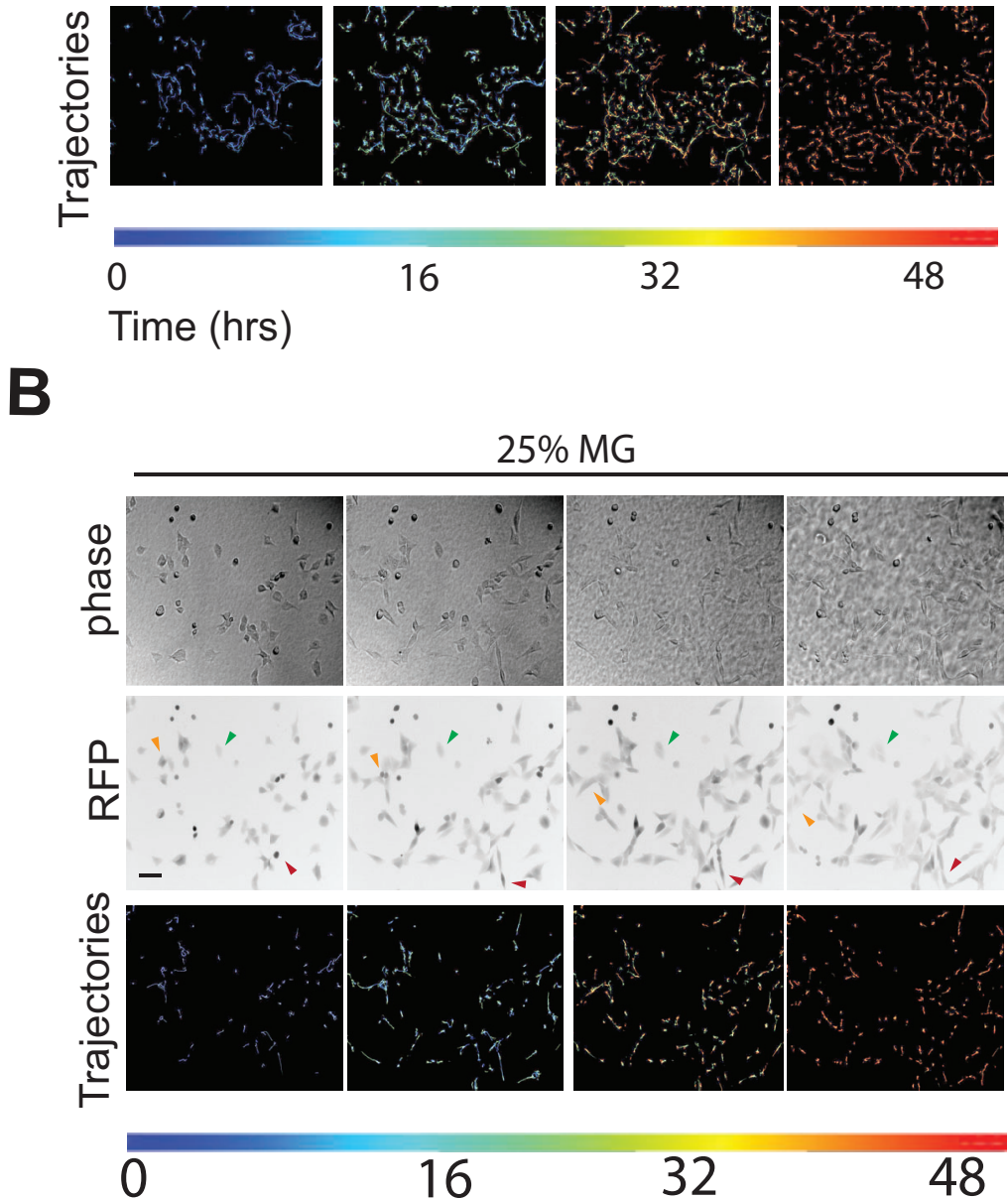
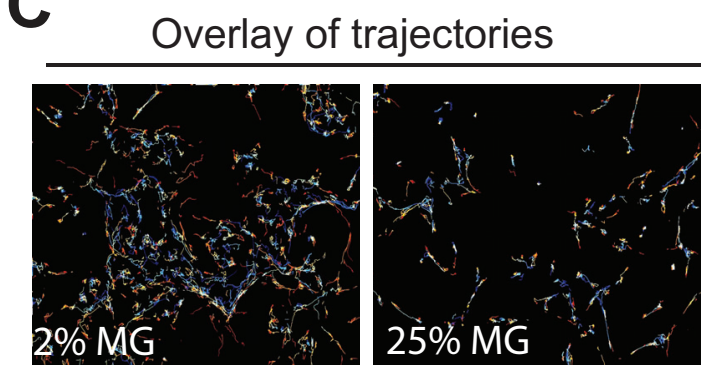

D

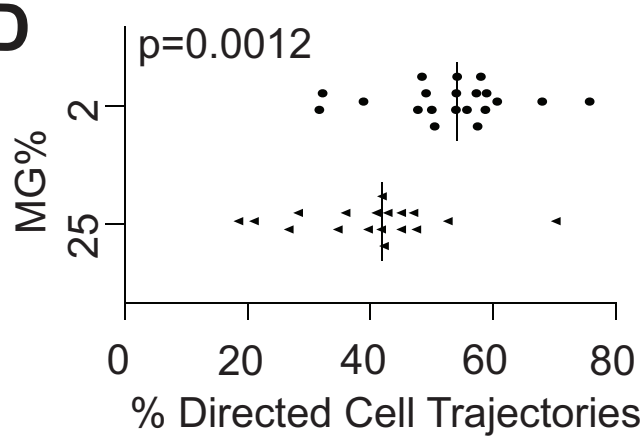

E

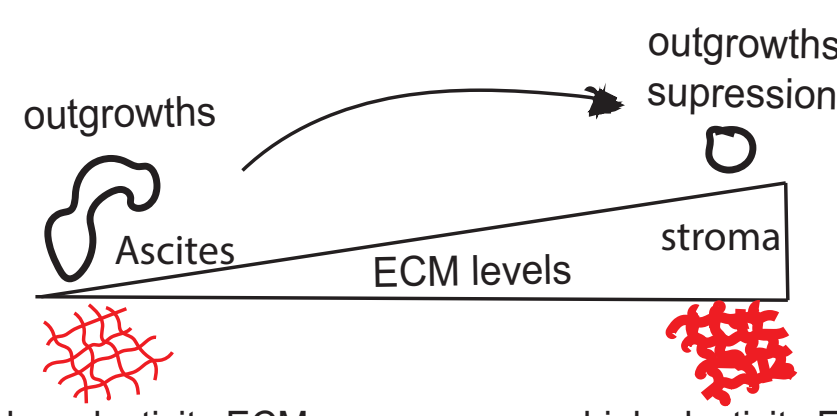

low elasticity ECM thin network fibers high elasticity ECM

thick network fibers

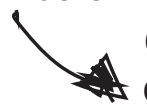

Ovarian Cancer cell proliferation cell motility

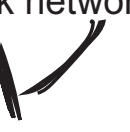

Time (hrs)

Figure 7. Elevation of ECM levels supresses cell translocation. (A) Representative phase contrast-, fluorescentand cell-trajectory images representing cell translocation within FNE-m-p53 cell monolayers overlayed with $2 \%$ or 25\% MG. (C) Represenative images of cell trajectories dynmics. (D) Distribution of \% classified cell trajectories within FNE-m-p53 cell monolayers overlayed with various concentrations of MG. 400-1000 cell trajectories were quantified within 9-10 fields of view representing each condition. p value was derived using Welch's t-test. (E) Graphical representation of key findings in this study. Bar $100 \mu \mathrm{m}$. 

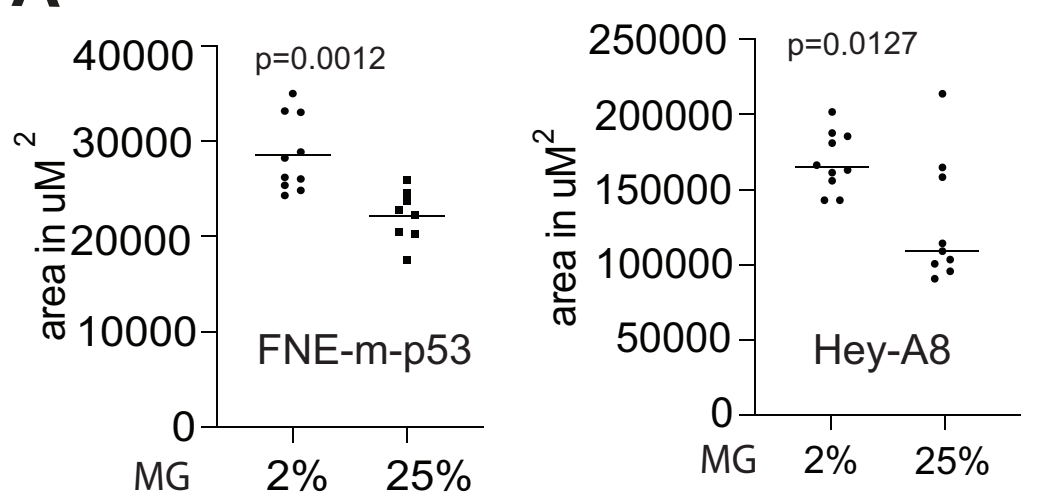

ECM encapsulation of 2D cultures Matrigel

Flat culture surface

Cells

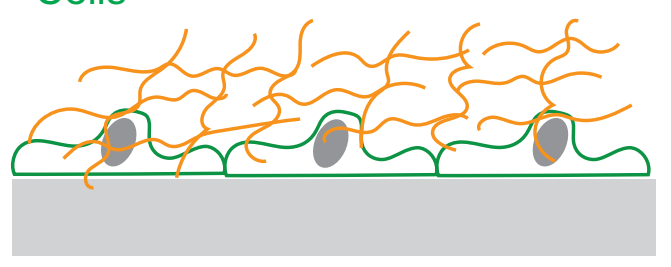

D FNE-m-p53-GFP

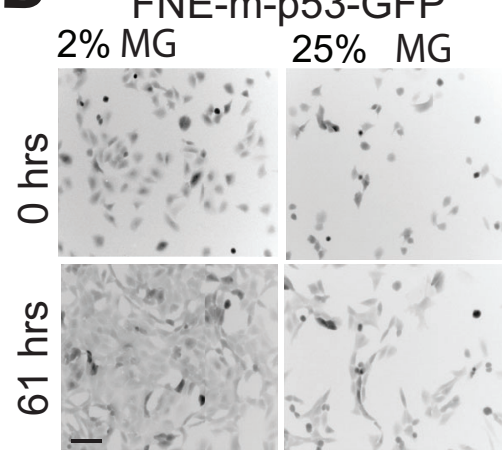

GFP

F

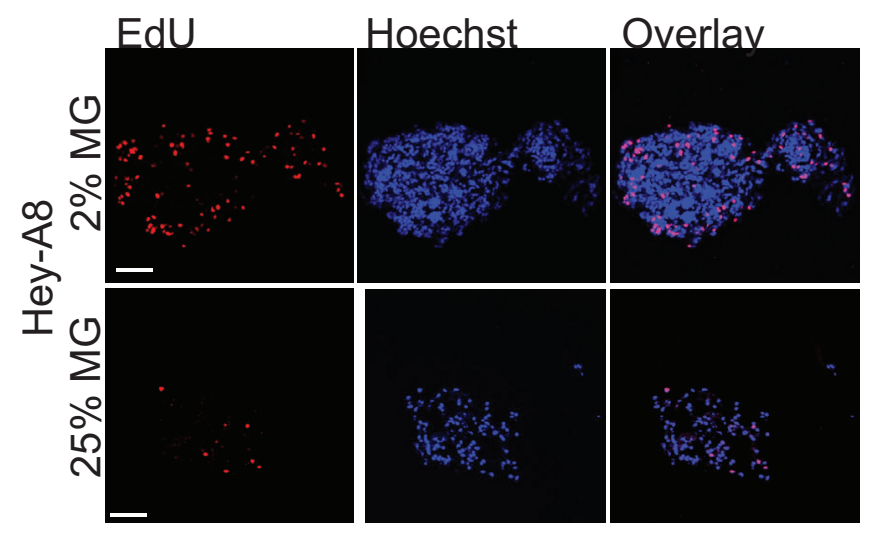

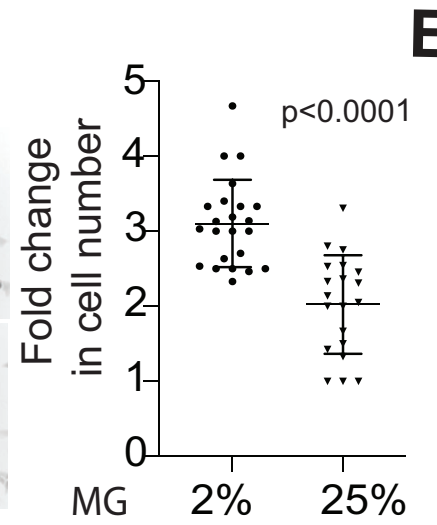

E
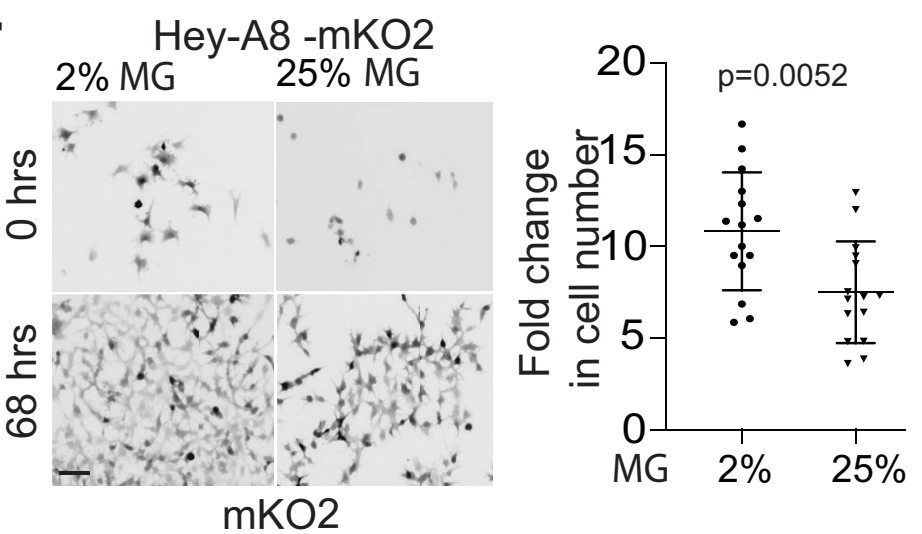

G
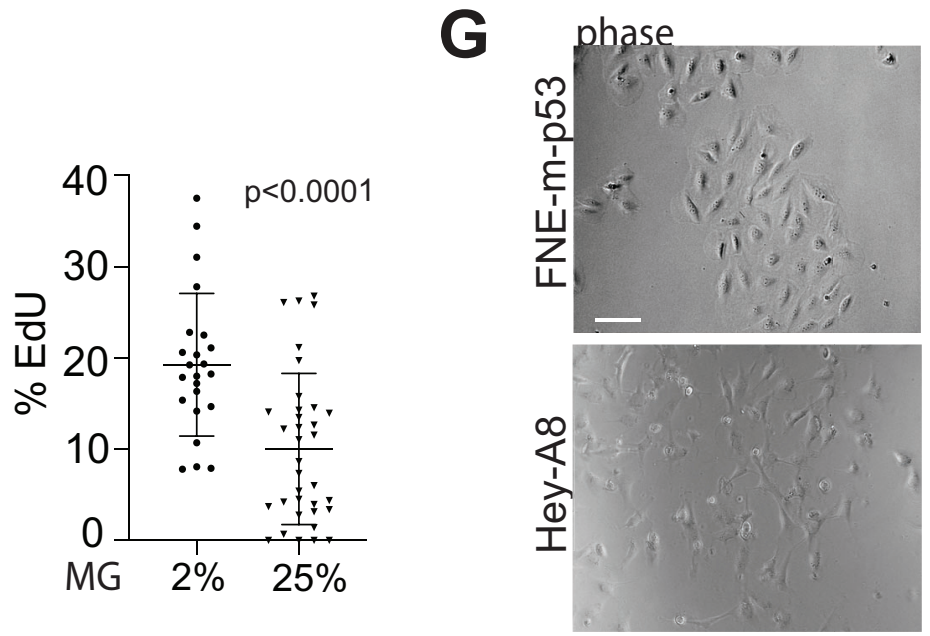

S.Figure 1. Elevation of ECM suppresses FNE-m-p53 and HEY-A8 cell proliferation. Quantification of an area in (A) FNE-m-p53 or (B) Hey-8A suspended cell clusters reconstituted with $2 \%$ or $25 \%$ MG. Dots represent individual cell cluster structure measured 9 days after starting the culture. (C) Cartoon representation of the experimental design for ECM encapsulation of cell monolayers. Representative fluorescent images of (D)

FNE-m-p53 or (E) Hey-A8 cells expressing RFP grown under condition of $2 \%$ or $25 \%$ MG encapsulation. Dot plots represent fold change increase in cell number over time. Each dot corresponds to one field of view. $(F)$ Representative fluorescent images of EdU and Hoechst in suspended Hey-A8 cells and reconstituted with $2 \%$ and $25 \%$ MG. Cell structures were processed 9 days after starting the culture. Dot plot represent the fraction of EdU positive cells. Each dot corresponds to one structure. Welch's t-test was used to compute statistical difference. (G) Phase contrast images of FNE-m-p53 and Hey-A8 cells grown as 2D adherent cultures. Scale bars are $100 \mu \mathrm{m}$. 